\title{
Entanglement spreading after a geometric quench in quantum spin chains
}

\author{
Vincenzo Alba ${ }^{1}$ and Fabian Heidrich-Meisner ${ }^{1}$ \\ ${ }^{I}$ Department of Physics and Arnold Sommerfeld Center for Theoretical Physics, \\ Ludwig-Maximilians-Universität München, D-80333 München, Germany
}

(Dated: July 28, 2018)

\begin{abstract}
We investigate the entanglement spreading in the anisotropic spin-1/2 Heisenberg $(X X Z)$ chain after a geometric quench. This corresponds to a sudden change of the geometry of the chain or, in the equivalent language of interacting fermions confined in a box trap, to a sudden increase of the trap size. The entanglement dynamics after the quench is associated with the ballistic propagation of a magnetization wavefront. At the free fermion point ( $X X$ chain), the von Neumann entropy $S_{A}$ exhibits several intriguing dynamical regimes. Specifically, at short times a logarithmic increase is observed, similar to local quenches. This is accurately described by an analytic formula that we derive from heuristic arguments. At intermediate times partial revivals of the short-time dynamics are superposed with a power-law increase $S_{A} \sim t^{\alpha}$, with $\alpha<1$. Finally, at very long times a steady state develops with constant entanglement entropy, apart from oscillations. As expected, since the model is integrable, we find that the steady state is non thermal, although it exhibits extensive entanglement entropy. We also investigate the entanglement dynamics after the quench from a finite to the infinite chain (sudden expansion). While at long times the entanglement vanishes, we demonstrate that its relaxation dynamics exhibits a number of scaling properties. Finally, we discuss the short-time entanglement dynamics in the $X X Z$ chain in the gapless phase. The same formula that describes the time dependence for the $X X$ chain remains valid in the whole gapless phase.
\end{abstract}

\section{INTRODUCTION}

The recent extraordinary progress achieved with trapped cold-atomic gases experiments has boosted a renewed theoretical interest in the dynamics of isolated quantum manybody systems out-of-equilibrium [1-3]. Highly-investigated topics include the relaxation dynamics [4-9], thermalization $[5,10]$ in out-of-equilibrium steady states, and transport properties [11-13]. A popular out-of-equilibrium experiment is the so-called quantum quench, in which a system is initially prepared in the ground state of a many-body quantum Hamiltonian, and a non-trivial unitary dynamics is then induced by changing instantaneously (i.e., "quenching") one (or many) control parameters. Depending on whether this change happens locally or in the whole system, the quench falls into the class of local or global quenches, respectively.

Entanglement measures are nowadays accepted as useful tools to extract universal properties of quantum many-body systems, both in and out-of-equilibrium [14-20]. Considering a bipartition of a system that is in a pure state $|\psi\rangle$ into parts $A$ and $B$, a standard measure of their mutual entanglement is the von Neumann entropy $S_{A}$

$$
S_{A}=-\operatorname{Tr} \rho_{A} \log \rho_{A} .
$$

Here $\rho_{A}$ is the reduced density matrix for $A$, obtained after tracing part $B$ from the full density matrix $\rho \equiv|\psi\rangle\langle\psi|$.

The real-time entanglement dynamics (and that of related quantities) after a quantum quench has been intensively investigated in recent years, both analytically (using conformal field theory [21-23] and for exactly solvable models [24-36]) and numerically [37-46]. The nature of the quench is strikingly reflected in the time dependence of the von Neumann entropy: while local quenches are associated with a logarithmic growth [21, 23, 24], a more dramatic (linear) behavior is observed in global quenches [22, 37, 38, 47]. This is related to the different excess energy density, measured with respect to the ground-state energy of the post-quench Hamiltonian. This excess energy remains finite in the global quench protocol, whereas it vanishes in the local one, upon increasing the system size. We mention that a scheme for measuring entanglement dynamics in cold-atomic gases experiments has recently been proposed in Ref. 48, 49.

In our work we focus on a situation that is intermediate between a local and a global quench, considering the real-time entanglement dynamics following an instantaneous change of the geometry or the size of the system, the so-called geometric quench, as discussed in Ref. 50. To be specific, we study the spin-1/2XXZ chain in the gapless phase. The quench protocol is described as follows (cf. Fig. 1): initially two chains $A$ and $B$ are prepared in the ground state of the $X X Z$ model in the sector with zero and maximum magnetization (i.e., fully polarized), respectively. The unitary dynamics under the $X X Z$ Hamiltonian is then induced by connecting the two chains. Alternatively, after mapping the $X X Z$ chain onto a system of interacting fermions confined in a box trap (i.e., chain $A$ ), the geometric quench is equivalent to suddenly increasing the trap size. Notice that this is similar to the socalled sudden expansion protocol used in cold-atomic gases experiments [11-13, 51], in which particles are released from the trap and expand in an empty lattice. This sudden expansion has been studied theoretically in, e.g., Refs. 44, 52-61.

Clearly, as the two chains are prepared in their respective ground states, the post-quench dynamics is induced by a "defect" at the interface between $A$ and $B$, which is a distinctive feature of local quenches. On the other hand, the excess energy density is finite, as in global quenches. Notice that the initial state after the quench is of the "domain wall" type (i.e., spatially inhomogeneous), and the ensuing out-of-equilibrium dynamics has been at the focus of many recent theoretical studies [33, 34, 62-73]. For instance, the state $\left|m_{A}\right\rangle \otimes\left|m_{B}\right\rangle$, with $m_{A}$ and $m_{B}$ being the total magnetization in chain $A$ and $B$, respectively, provides a ba- 
sic setup for studying transport-related questions and nonequilibrium steady-state properties such as the conditions for ballistic or diffusive dynamics in integrable many-body systems in one dimension $[74,75])$. In particular, the sub-class of initial states with $m_{A}=-m_{B} \equiv m$ has been extensively studied $[33,34,62,64-66,68,69,72,73]$. The initial state in our work corresponds to choosing $m_{A}=0$ and $m_{B}=L_{B} / 2$. Interestingly, in the situation with $m_{A}=-m_{B}$ it has been found that the magnetization dynamics during the domain wall melting is ballistic close to the free fermion point, super-diffusive at the isotropic point, and diffusive in the gapped phases [64]. While entanglement dynamics from domain wall initial states is interesting as such, it is also important for the simulability of quench dynamics using matrixproduct states based methods, such as DMRG (Density Matrix Renormalization Group) [76-78]. For a discussion of transport and local quenches in spin chains in non-equilibrium for other initial conditions, see Ref. [79-86]. We should also mention that transport and entanglement properties have also been studied in the dynamics induced by local impurities [27, 30, 39, 41, 87].

Summary of the results. - In this work we fully characterize the entanglement spreading after a generic geometric quench, focusing on the entanglement entropy between $A$ and $B$. The spreading of information (and the related entanglement increase) is associated with the propagation of an extended magnetization wavefront. The two edges of the front expand ballistically in the $A$ and $B$ parts of the chain, with two different velocities. These coincide at the free-fermion point ( $X X$ chain, i.e., vanishing anisotropy), where the wavefront propagates symmetrically. For the $X X$ chain the full magnetization profile, at any time after the quench, is obtained analytically, using a semiclassical reasoning and free-fermionic techniques (as in [88]). For the $X X Z$ chain, although we do not derive analytically the full magnetization profile, we provide an approximate expression describing the central region of the wavefront.

The entanglement evolution exhibits several dynamical behaviors at different time scales. For the $X X$ chain all these dynamical regimes are thoroughly investigated, exploiting the mapping to free fermions. At short times, the von Neumann entropy increases logarithmically, as in a local quench. Although the well-known conformal field theory (CFT) result for the local quench $[21,23]$ does not apply, we provide a heuristic extension of this result to our case, which accurately reproduces the entanglement dynamics. One remarkable consequence is that the entanglement dynamics, apart from a sizedependent shift, is described by a scaling function $f_{s}(y)$, with $y \equiv t / L_{A}$ ( $t$ is the time after the quench and $L_{A}$ the size of part $A$ ). We numerically demonstrate that the same scaling holds true in the interacting case.

At intermediate times the entanglement entropy exhibits revivals of the short-time dynamics, superposed with a powerlaw increase as $S_{A} \sim t^{\alpha}$ (apart from possible multiplicative logarithmic corrections). We numerically extract the exponent $\alpha$, finding $\alpha<1$. This suggests that the geometric quench cannot be thought of as a simple superposition of a logarithmic (i.e., local-quench like) and a linear (as in global (a)

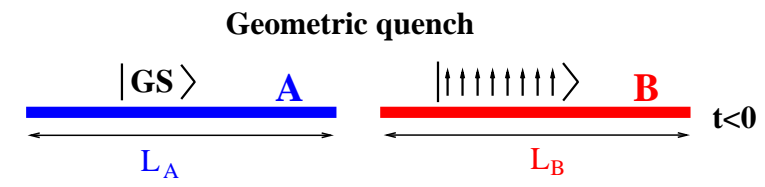

(b)

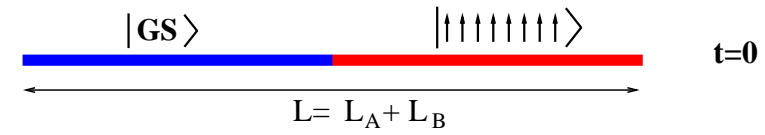

(c)

Local quench

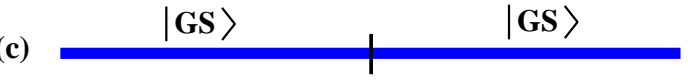

FIG. 1: (a), (b) Geometric quench in $X X Z$ spin chain: quench setup for open boundary conditions. (a) $t<0$ : Two independent chains ( $A$ and $B$ ) of length $L_{A}$ and $L_{B} \equiv L-L_{A}$ are prepared in the ground state of the $X X Z$ chain in the sectors with zero and maximal magnetization, respectively. (b) $t=0: A$ and $B$ are glued together. Here we consider geometric quenches with several aspect ratios $\omega \equiv$ $L_{A} / L$, i.e., $0 \leq \omega \leq 1$, focusing on the entanglement between $A$ and $B$. (c) Example of typical local quench protocol: the initial state at $t=0$ is obtained connecting two identical chains prepared in the ground state at zero magnetization.

quenches) behaviors.

At long times the system reaches an out-of-equilibrium steady state, and the entropy oscillates around a stationary value. The steady state is non thermal and shows features of the initial Fermi surface in part $A$ of the chain. Finally, in spite of the non-thermal nature of the steady state, we demonstrate that the entanglement entropy is extensive, and its stationary value can be determined analytically. Similar results have been found in Ref. 33 for the quench from the state $|-m\rangle \otimes|m\rangle$, while the constraints put on steady states due to integrability for a similar set-up and hard-core bosons, which map on the $X X$ model, were discussed in a seminal paper by Rigol et al. [89].

We also discuss the information spreading after the quench from a finite to the infinite chain. In the framework of trapped interacting fermions, this corresponds to removing the trap completely, i.e., to the so-called sudden expansion. While the entanglement entropy vanishes asymptotically (i.e., at large times), its relaxation dynamics shows unexpected scaling behaviors. In particular, the entanglement dynamics is described by a scaling function $f_{\ell}(z)$ with $z \equiv t / L_{A}^{2}$. Furthermore, $f_{\ell}(z)$ exhibits an intriguing structure: while at $z \ll 1$ one has $f_{\ell}(z) \sim-\log (1 / z)$, a crossover to the behavior $1 / z(1-\log (1 / z))$ occurs at $z \sim 1$. Similar scaling behaviors have been observed in the entanglement dynamics of non-interacting fermions in continuous space released from a trap [90-92].

Finally, by means of tDMRG [93, 94] (time-dependent Density Matrix Renormalization Group) simulations, we investigate the role of interactions on the short-time entanglement dynamics, focusing on the $X X Z$ spin chain. Our main result is that the same formula used for the $X X$ chain remains valid. Interestingly, since the excitations forming the wavefront propagate in the two parts of the chain with different 
velocities, we find that the entanglement spreading rate is not a trivial function of the spinon velocity.

Outline.- This paper is organized as follows. In Sec. II we introduce the $X X Z$ spin chain and the geometric quench protocol. Sections III, IV, V, VI, and VII are devoted to the $X X$ chain. In Sec. III we investigate the magnetization wavefront expansion. An overview of the entanglement dynamics after the quench is given in Sec. IV, while the short-time behavior is discussed in detail in Sec. V. In Sec VI we characterize the entanglement properties in the steady state. The infinite-chain quench is then discussed in Sec. VII. Finally, in Sec. VIII we investigate the short-time entanglement dynamics in the $X X Z$ model, while a summary is provided in Sec. IX.

\section{MODEL \& QUENCH PROTOCOL}

\section{A. The spin- $1 / 2 X X Z$ spin chain}

The open $X X Z$ spin chain of length $L$ is defined by the Hamiltonian

$$
\mathcal{H}=\frac{J}{2} \sum_{i=1}^{L-1}\left(S_{i}^{+} S_{i+1}^{-}+\text {h.c. }\right)+J \Delta \sum_{i=1}^{L-1} S_{i}^{z} S_{i+1}^{z} .
$$

Here $S_{i}^{ \pm} \equiv S_{i}^{x} \pm i S_{i}^{y}, S_{i}^{z} \equiv S_{i}^{z}$ are spin- $1 / 2$ operators acting at site $i$ of the chain, and $\Delta$ the so-called anisotropy (we set $J=1$ in Eq. (2)). For a periodic chain an extra term in Eq. (2) connects sites 1 and $L$. The ground-state phase diagram of the $X X Z$ chain exhibits a gapless spin-liquid phase at $-1<$ $\Delta \leq 1$, while it is gapped at $|\Delta|>1$. At $\Delta=0(X X$ chain) the $X X Z$ chain reduces to a free-fermionic model (cf. Appendix A for more details) [95].

The low-energy spectrum of Eq. (2) is linear in the spin liquid phase, and it is described (along with other low-energy properties) by a conformal field theory (CFT) with central charge $c=1$. At sufficiently large $L$ one has [96,97]

$$
E_{\alpha}=L E_{\mathrm{bulk}}+E_{\mathrm{bound}}+\frac{\pi v_{s}}{L}\left(h_{\alpha}-\frac{c}{24}\right)+\mathcal{O}\left(L^{-2}\right),
$$

with $E_{\alpha}$ being the energy of a low-lying excitation (labeled by $\alpha \in \mathbb{N}$ ) of Eq. (2). In Eq. (3), $E_{\text {bulk }}$ and $E_{\text {bound }}$ are the usual bulk (extensive) and a boundary (in presence of non-periodic boundary conditions) contributions, $c$ is the central charge (here $c=1$ ), and $v_{s}$ the spinon velocity. Finally, $h_{\alpha}$ are the scaling dimensions of the operators (both primary operators and their descendants $[98,99])$ appearing in the CFT. In particular, $\alpha=0$ (with $h_{\alpha}=0$ ) corresponds to the ground-state energy $E_{0}$. Finite-size deviations from the linear dispersion are accounted for by the $\mathcal{O}\left(1 / L^{2}\right)$ term. Notice that Eq. (3) can be thought of as the spectrum of an effective Hamiltonian $\mathcal{H}_{C F T}$. The two energy scales set by the terms $\sim 1 / L$ and $\sim 1 / L^{2}$ in Eq. (3) imply the existence of two typical time scales $t_{s}^{*} \sim L / v_{s}$ (short times) and $t_{\ell}^{*} \sim L^{2} / v_{s}$ (long times) (here all lengths are measured in units of the lattice constant $a=1$ ). We anticipate that the existence of $t_{s}^{*}$ and $t_{\ell}^{*}$ will be strikingly reflected in the entanglement dynamics after the quench.

\section{B. Quench protocol and observables}

The geometric quench protocol for the $X X Z$ spin chain with open boundary conditions (the generalization to periodic boundary conditions is straightforward) is depicted in Fig. 1. At time $t<0$ (Fig. 1 (a)) two disconnected chains $A$ and $B$ (of respective lengths $L_{A}$ and $L_{B}$ ) are prepared in the ground state $|G S\rangle$ of Eq. (2) and in the fully polarized (ferromagnetic) state $|F\rangle \equiv|\uparrow \uparrow \ldots \uparrow\rangle$, respectively. The latter is an eigenstate of Eq. (2) at any $\Delta$, with eigenenergy $E \equiv\langle\mathcal{H}\rangle=(L-1) \Delta / 4$. At $\Delta \gg-1$ in the gapless phase, which is the region of interest here, $|F\rangle$ is in the highenergy part of the spectrum of Eq. (2), and at the isotropic point $(\Delta=1)$ it is the highest-energy eigenstate.

At $t=0$ the two chains $A$ and $B$ are connected to form a new one of total length $L \equiv L_{A}+L_{B}$ (cf. Fig. 1 (b)). The initial quantum state $\left|\Psi_{\text {init }}\right\rangle$ after the quench exhibits a steplike (or "domain wall") magnetization profile (with $\left\langle S_{i}^{z}\right\rangle=0$ and $\left\langle S_{i}^{z}\right\rangle=1 / 2$ for $i \in A$ and $i \in B$ respectively). It is useful to introduce the aspect ratio $0 \leq \omega \leq 1$ as

$$
\omega \equiv \frac{L_{A}}{L}
$$

Finally, at $t>0$ the chain evolves unitarily under Eq. (2) since $\left|\Psi_{\text {init }}\right\rangle$ is not an eigenstate. In this work we focus on the realtime dynamics of the von Neumann entropy $S_{A}$ between $A$ and $B$.

\section{Geometric vs local quench}

It is interesting to compare the geometric quench with a local quench $[21,23,24,27,29,64,100,101]$. A typical local quench is illustrated in Fig. 1 (c): the initial state $\left|\Psi_{\text {init }}\right\rangle$ at $t=0$ is now obtained by "gluing" together two identical copies of the ground state as $\left|\Psi_{\text {init }}\right\rangle \equiv|G S\rangle \otimes|G S\rangle$ (which implies that $\omega=1 / 2$ ).

Clearly, the excess energy density, which is defined as $\delta_{e} \equiv$ $\left|\left\langle\Psi_{\text {init }}|\mathcal{H}| \Psi_{\text {init }}\right\rangle-E_{0}\right| / L$, vanishes in the local quench $\left(\delta_{e} \sim\right.$ $\mathcal{O}(1 / L)$, cf. Eq. (3)) in the limit $L \rightarrow \infty$. Oppositely, in the geometric quench, due to the typically large energy of chain $B$, one has $\delta_{e} \sim \mathcal{O}(1)$. As a consequence, while only few low-lying excitations (cf. Eq. (2)) play a role in the dynamics after a local quench, this is certainly different in the geometric quench.

In the CFT framework the initial quantum state $\left|\Psi_{\text {init }}\right\rangle$ can be decomposed (in analogy with the standard decomposition in the eigenbasis of Eq. (2)) as

$$
\left|\Psi_{\text {init }}\right\rangle=\sum_{a} c_{a}\left|\phi_{a}\right\rangle
$$

where the sum runs over both primary and descendants fields $\phi_{a}$ of the CFT. In principle Eq. (5) provides all the necessary information about the post-quench dynamics, after time evolving each eigenstate $\left|\phi_{a}\right\rangle$ of $\mathcal{H}_{C F T}$ with $e^{-i \mathcal{H}_{C F T} t}$.

However, the coefficients $c_{a}$ in Eq. (5) are not easy to calculate for a generic initial state. For the local quench this is pos- 
sible because only one operator (the identity) and its descendants enter in the expansion Eq. (5) [23]. This is related to the fact that $\left|\Psi_{\text {init }}\right\rangle$ has substantial overlap only with the ground state of Eq. (2). A prominent consequence is that the entanglement entropy dynamics after a local quench shows perfect revivals (apart from scaling corrections) for $t \sim n t_{s}^{*}, n \in \mathbb{N}$, at least up to the time $t \sim t_{\ell}^{*}$, at which the CFT description is no longer valid, cf. Eq. (3) [23]. Conversely, this will be strikingly different for the geometric quench (cf. Sec. IV).

\section{Entanglement dynamics after a local quench}

Here we briefly review the CFT result for the entanglement dynamics after the local quench in Fig. 1 (c) [21, 23]. The real-time dynamics of the von Neumann entropy depends only on the central charge $c$, the boundary conditions, and the spinon velocity $v_{s}$. The result reads [23, 27]

$$
S_{A}(t)=\nu \frac{c}{3} \log \left|\frac{L_{A}}{\pi} \sin \frac{\nu \pi v_{s} t}{2 L_{A}}\right|+k_{\nu} .
$$

Here $\nu=1,2$ are for open (obc) and periodic boundary conditions (pbc), respectively, and $k_{\nu}$ is a non-universal constant. The analog of Eq. (6) for aspect ratios $\omega \neq 1 / 2$ is also known [23]. It is useful to rewrite Eq. (6) as

$$
S_{A}(t)=\nu \frac{c}{3} \log |\sin (\pi y)|+\nu \frac{c}{3} \log \left(\frac{L_{A}}{\pi}\right)+k_{\nu}
$$

Here $y$ is the rescaled time $y \equiv \nu \pi v_{s} t /\left(2 L_{A}\right)$. In Eq. (7) it is apparent that the entropy dynamics is described by a scaling function of $y$, apart from the shift $\nu c / 3 \log \left(L_{A}\right)$. Interestingly, the latter resembles the equilibrium ground-state entropy for a block of size $L_{A}$ embedded in an infinite chain [14-17]. A similar scaling will hold for the entanglement dynamics at short times after the geometric quench (cf. Sec. V). In the limit $t / L_{A} \ll 1$ (short times), Eq. (6) reduces to [21]

$$
S_{A}(t)=\nu \frac{c}{3} \log t+k_{\nu}
$$

A similar logarithmic behavior (as in Eq. (6) and Eq. (8)) has been observed in the entanglement dynamics induced by local impurities or perturbations in spin or particle densities [27, 30, $41,80]$.

\section{MAGNETIZATION WAVEFRONT AFTER THE QUENCH}

In this section we discuss the real-time dynamics of the magnetization profile $\left\langle S_{i}^{z}\right\rangle$ after a geometric quench. Here $\langle\cdot\rangle$ denotes the expectation value with respect to the post-quench wavefunction. We focus on the open $X X$ chain $(\Delta=0$ in Eq. (2)). At any $i, t,\left\langle S_{i}^{z}\right\rangle$ can be computed analytically exploiting the mapping to free fermions (see Appendix A).

Figure 2 shows $\left\langle S_{i}^{z}\right\rangle$ versus $1 \leq i \leq L$ at several times (denoted by different symbols in the figure) after the geometric quench with $\omega \equiv L_{A} / L=1 / 3$ and fixed $L_{A}=60$. At $t=0$,

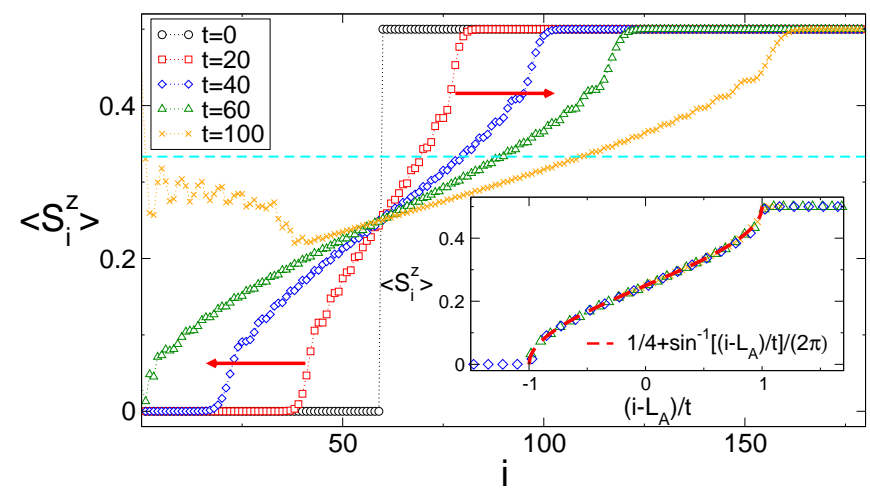

FIG. 2: Magnetization wavefront after the geometric quench with aspect ratio $\omega \equiv L_{A} / L=1 / 3$ (cf. Fig. 1) in the open $X X$ chain: local magnetization $\left\langle S_{i}^{z}\right\rangle$ at site $0 \leq i<3 L_{A}$ in the chain. Data are exact results for $L_{A}=60$ and several times. At $t=0$ a step-like profile is present. At $t>0$ a wavefront forms propagating symmetrically (with $v_{s}=1$ ) in parts $A$ and $B$ (see horizontal arrows). The dashed line is the flat profile expected on average at $t \rightarrow \infty$. At $t=L_{A} / v_{s}$ the wavefront is reflected at the (left) boundary of the chain. Inset: rescaled dynamics, $\left\langle S_{i}^{z}\right\rangle$ versus $\left(i-L_{A}\right) / t$. All data for different times collapse on the same scaling function (dashed line).

a domain-wall profile is present. A magnetization wavefront develops at $t>0$ (as the domain wall "melts") with its left and right edges propagating ballistically with the same velocity $v=v_{s}=1$, with $v_{s}$ being the spinon velocity, in part $A$ of the chain. At $t=L_{A} / v_{s}$, a perfect reflection of the left wavefront edge occurs at the left boundary of the chain. Finally, at large times translational invariance is restored, and a stationary behavior sets in with uniform magnetization (dashedhorizontal line in the figure).

The ballistic nature of the wavefront dynamics is further supported by the data shown in the inset of Fig. 2, where we plot $\left\langle S_{i}^{z}\right\rangle$ versus the rescaled variable $\left(i-L_{A}\right) / t$. Remarkably, all data at different times and positions collapse on the same scaling curve. This curve can be obtained analytically using a semiclassical reasoning that was also applied in Refs. [33, 34, $62,64,88,102]$ for the quench with initial state $|-m\rangle \otimes|m\rangle$. The result reads

$$
\left\langle S_{i}^{z}(t)\right\rangle=\frac{1}{4}+\frac{1}{2 \pi} \sin ^{-1}\left[\frac{i-L_{A}}{v_{s} t}\right]
$$

and is included in Fig. 2 as dashed line. Interestingly, the central region of the profile, at $\left|\left(i-L_{A}\right) /\left(v_{s} t\right)\right| \ll 1$, shows a linear dependence on $\left(i-L_{A}\right) / t$ :

$$
\left\langle S_{i}^{z}(t)\right\rangle \approx \frac{1}{4}+\frac{i-L_{A}}{v_{s} t}
$$

It is natural to expect that Eq. (10) remains valid in the interacting case (i.e., nonzero anisotropy), after taking into account the renormalization, due to interactions, of the the spinon velocity $v_{s}$ (see Sec. VIII A for a numerical check of Eq. (10) in the $X X Z$ spin chain). 
(a) short times

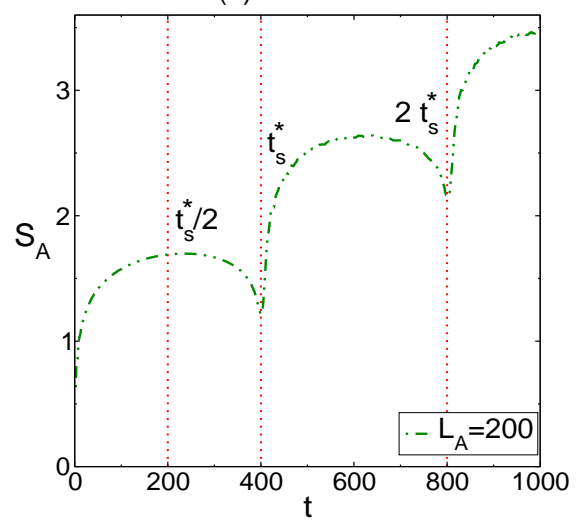

(b) semiclassical interpretation

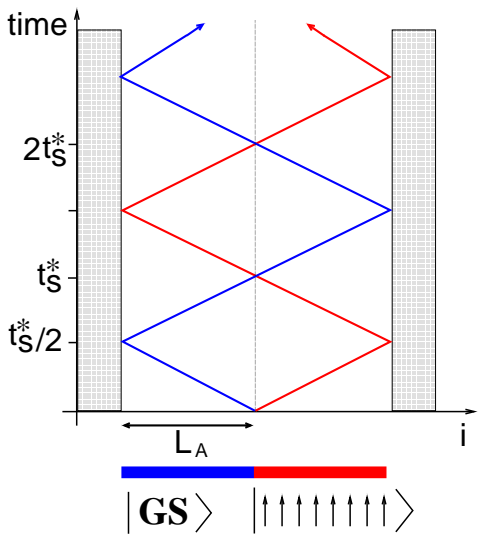

(c) long times

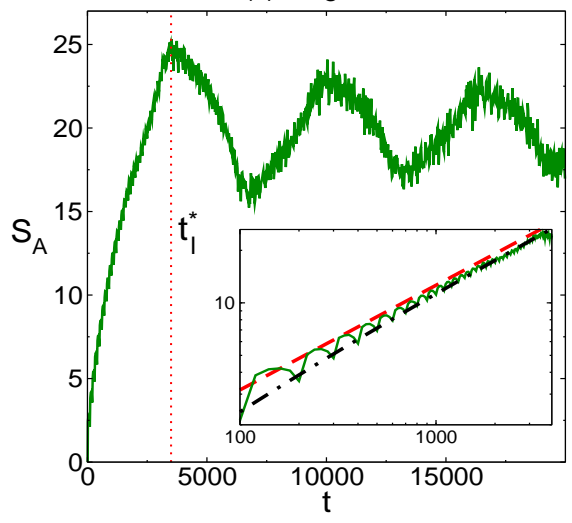

FIG. 3: Entanglement spreading after the geometric quench with aspect ratio $\omega \equiv L_{A} / L=1 / 2$ (cf. Fig. 1 ) in the open $X X$ chain. (a) von Neumann entropy (dashed-dotted line) $S_{A}$ for part $A$ of the chain as a function of time: exact results for $L_{A}=200,0 \leq t \leq t_{s}^{*}$ (short times), with $t_{s}^{*} \equiv 2 L_{A} / v_{s}$ where $v_{s}=1$ is the spinon velocity. Dotted vertical lines mark the times $t=t_{s}^{*} / 2, t_{s}^{*}, 2 t_{s}^{*}$. (b) Semiclassical interpretation: entanglement growth is understood in terms of the ballistic propagation (with velocity $v_{s}=1$ ) of free effective excitations (the lines denote their trajectories). These are created at $t=0$ at the interface between $A$ and $B$. At $t=t_{s}^{*} / 2$, perfect reflection at the boundary of the chain occurs. Entanglement "jumps" at $t=m t_{s}^{*}, m=1,2 \ldots$ correspond to excitations crossing the center of the chain. (c) Long-time behavior: at $t \sim t_{\ell}^{*} \equiv L_{A}^{2} / v_{s}$ (i.e., after $\mathcal{O}\left(L_{A}\right)$ crossings) the system reaches a steady state with constant entropy (apart from superimposed oscillations). Inset: approach to the steady-state entanglement (data for the same parameters as in the main figure and $0 \leq t \leq t_{\ell}^{*}$ ). A logarithmic scale is used on both axes. The dashed line is $S_{A} \sim t^{\alpha}$, with $\alpha \approx 0.6$, whereas the dashed-dotted one is $S_{A} \sim t^{1 / 2} \log (t)$.

\section{ENTANGLEMENT SPREADING IN FREE SYSTEMS: OVERVIEW}

We now turn to the real-time dynamics of the entanglement entropy $S_{A}$ between the two parts $A$ and $B$ of the chain. Here we consider the open $X X$ chain, restricting ourselves to an aspect ratio of $\omega=1 / 2$ (see Fig. 1). The calculation of the entanglement entropy after the geometric quench in the $X X$ chain is outlined in Appendix C.

Clearly, at $t=0, A$ is in a pure state, implying $S_{A}=0$. Exact numerical data at $t>0$ after the quench (with fixed $L_{A}=200$ ) are shown in Fig. 3 [dashed-dotted line in panels (a) and (c)]. $S_{A}$ exhibits different behaviors at different time scales. At short times $t \leq t_{s}^{*} / 2$, with $t_{s}^{*} \equiv 2 L_{A} / v_{s}$, (cf. Fig. 3 (a)) the entanglement entropy grows logarithmically as in a local quench. In the time-interval $t_{s}^{*} / 2 \lesssim t \leq t_{s}^{*}$, it slightly decreases reflecting the finite size of part $A$.

At intermediate times $t_{s}^{*}<t \leq t_{\ell}^{*}$ (cf. Fig. 3 (c)), $S_{A}$ grows with a power law (cf. the inset in Fig. 3 (c)). A fit to $S_{A} \sim t^{\alpha}$ yields $\alpha \approx 0.6$ (dashed line in the inset). However, we should stress that the data are also compatible with the behavior $S_{A} \sim t^{1 / 2}|\log (t)|$ (dashed-dotted line in the inset). A similar power-law increase of the entanglement entropy has been observed in quantum quenches in quasicrystals [103]. Interestingly, partial revivals of the short-time dynamics are superposed with the power-law growth, in contrast with the local quench, where perfect revivals occur, apart from scaling corrections [23].

The qualitative behavior of the entanglement can be understood in a semiclassical picture in terms of the ballistic propagation of the magnetization wavefront discussed in Sec. III. This is illustrated in Fig. 3 (b). The initial entanglement in- crease at $t>0$ corresponds to the two edges of the wavefront (red and blue lines in the figure) propagating with equal velocities in the two parts of the chain. At $t=t_{s}^{*} / 2$ the two edges are reflected at the physical boundaries. Finally, at $t=t_{s}^{*}$, a crossing of the two edge trajectories occurs. Every crossing at the later times $t=k t_{s}^{*}, k \in \mathbb{N}$, is reflected in an sudden increase in the von Neumann entropy (Fig. 3 (a)). A similar semiclassical picture [104] holds in the case of a local quench [22], where the entanglement growth is associated with the propagation of two "localized" defects [24, 100, 101].

At $t>t_{\ell}^{*} \sim L_{A}^{2} / v_{s}$, i.e., after $\mathcal{O}\left(L_{A}\right)$ crossings of the wavefront edge trajectories, (cf. Fig. 3 (b)), the system reaches a steady state and the von Neumann entropy oscillates around a stationary value. We anticipate that, since the model is integrable [89], the steady state is different from a thermal state, although its entanglement entropy is extensive (cf. Sec. VI).

\section{SHORT-TIME ENTANGLEMENT DYNAMICS}

In this section we focus on the short-time entanglement dynamics (i.e., at $t \leq t_{s}^{*}$, cf. Fig. 3 (a)). Here, in particular, we provide an analytic expression, which accurately describes the von Neumann entropy dynamics at short times $t \lesssim t_{s}^{*} \equiv \min \left(2 L_{A} /\left(\nu v_{s}\right), 2 L_{B} / \nu\right)$. We motivate this formula based on heuristic arguments. This result holds irrespective of the quench aspect ratio $\omega$ (see Fig. 4 (a)), since the spreading of information between $A$ and $B$ is associated with the propagation of the two wavefront edges (cf. Fig. 3 (b)) and part $B$ of the chain is prepared in the "vacuum" state.

Figure 4 shows $S_{A}(t)$ as a function of the rescaled time $\nu v_{s} t /\left(2 L_{A}\right) \leq 1$. Data are exact numerical results for the 


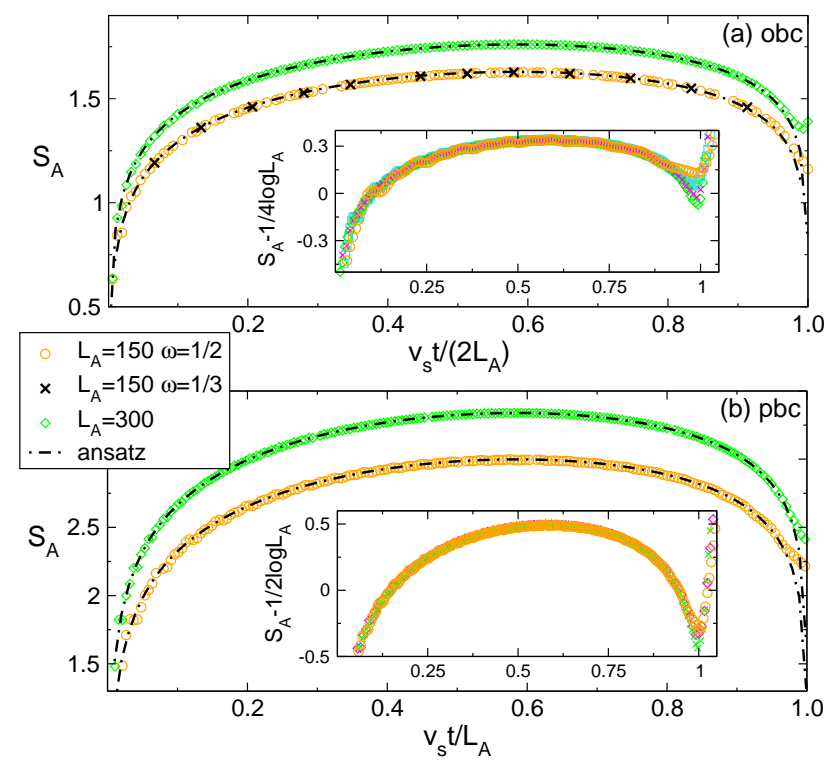

FIG. 4: von Neumann entropy $S_{A}$ for part $A$ after the geometric quench (with $\omega \equiv L_{A} / L=1 / 2,1 / 3$ ) in the $X X$ chain: shorttime behavior (at $t \leq t_{s}^{*} \sim L_{A} / v_{s}$, and $v_{s}=1$ the spinon velocity). (a) $X X$ chain with open boundary conditions, $S_{A}$ versus $v_{s} t /\left(2 L_{A}\right)$ (exact results for several sizes $\left.L_{A}\right)$. The crosses are data for $L=150$ and $\omega=1 / 3$. Dashed-dotted lines are one parameter fits to $S_{\text {ansatz }}$ (see Eq. (12)). Inset: shifted entropy, $S_{A}-1 / 4 \log L_{A}$ versus $v_{s} t /\left(2 L_{A}\right)$. Note the perfect data collapse for all chain sizes and times. (b) The same as in (a) for periodic boundary conditions: now $S_{A}$ is plotted versus $v_{s} t / L_{A}$. Dashed-dotted lines are fits to Eq. (12) (notice the dependence on boundary conditions). Inset: shifted entropy, $S_{A}-1 / 2 \log L_{A}$ versus $v_{s} t / L_{A}$.

$X X$ chain with either open or periodic boundary conditions (panels (a) and (b), respectively). For the sake of simplicity we restrict ourselves to a geometric quench with aspect ratio $\omega=1 / 2$. Motivated by the result for the local quench [21, 23, $25,27]$ Eq. (6) we have fitted the numerical data to

$$
S_{\text {ansatz }}(t)=\alpha_{\nu} \log (t)+\beta_{\nu} \log \left[L_{A} \sin \frac{\nu \pi v_{s} t}{2 L_{A}}\right]+\gamma_{\nu}
$$

where $\nu=1,2$ are for open and periodic boundary conditions, respectively, $v_{s}=1$ is the spinon velocity, and $\alpha_{\nu}, \beta_{\nu}, \gamma_{\nu}$ are fitting parameters. In Eq. (11) the first term is motivated by the fact that $S_{A}(t)$ is not symmetric under $t \rightarrow 2 L_{A} /\left(\nu v_{s}\right)-t$, i.e., left-right inversion (see Fig. 4), while the second one is similar to the local quench result Eq. (6). We have numerically found that $\beta_{\nu}=2 \alpha_{\nu}=\nu / 6$. Finally, we rewrite Eq. (11) as

$$
S_{\text {ansatz }}(t)=\frac{\nu}{6} \log \left|L_{A}^{\frac{3}{2}}\left(\frac{\nu v_{s} t}{2 L_{A}}\right)^{\frac{1}{2}} \sin \frac{\nu \pi v_{s} t}{2 L_{A}}\right|+k_{\nu}^{\prime},
$$

with $k_{\nu}^{\prime}$ a constant. In the limit $t / L_{A} \ll 1$ (short times), one obtains from Eq. (12)

$$
S_{A}(t)=\frac{\nu}{4} \log t+k_{\nu}^{\prime}
$$

which is different from the local-quench CFT result Eq. (8). A similar result is discussed in Ref. 105, where the ground-state
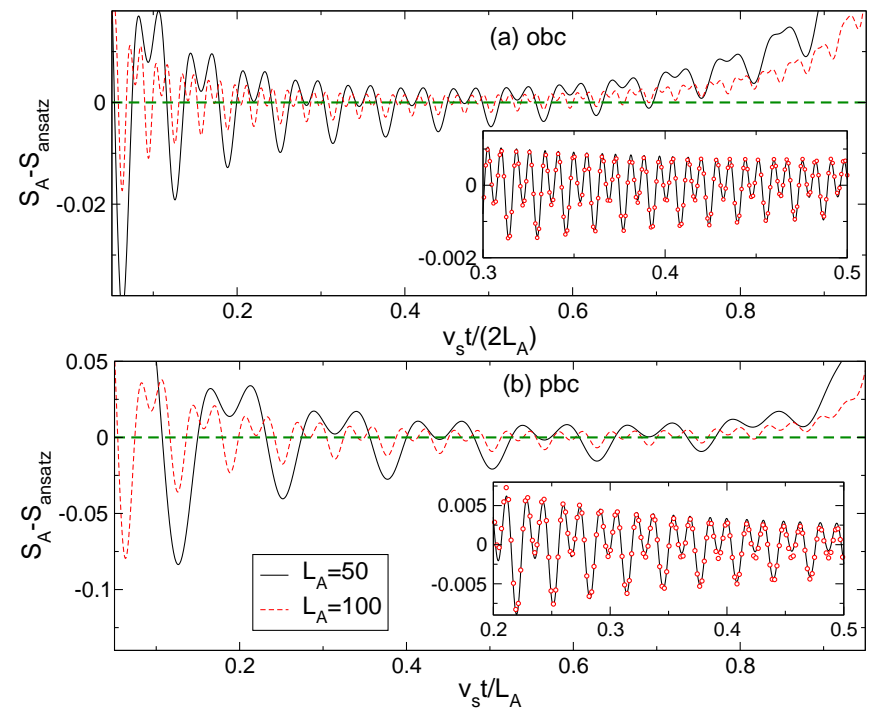

FIG. 5: Geometric quench ( $\omega \equiv L_{A} / L=1 / 2$, cf. Fig. 1$)$ in the $X X$ spin chain: short-time dynamics of the von Neumann entropy $S_{A}$ for part A of the chain. Deviations from $S_{\text {ansatz }}$ (see Eq. (12)) for (a) open and (b) periodic boundary condition. (a) $S_{A}-S_{\text {ansatz }}$ plotted versus $v_{s} t /\left(2 L_{A}\right)$. Data are exact results for $L_{A}=50,100$ (full and dashed line, respectively). Note that $k_{\nu}^{\prime}$ (see Eq. (12)) has been fitted and subtracted from the curves. Inset: same as in the main panel (circles are data for $L_{A}=100$ ), the continuous line is a fit to $\left.\left[a_{1} \cos (t)\right)+a_{2} \cos (2 t)\right] / t$. (b) Same as in (a) yet for periodic boundary conditions: $S_{A}-S_{\text {ansatz }}$ versus $v_{s} t / L_{A}$. Inset: same as in (a). The full line is a fit to $\left[a_{1} \cos (t)+a_{2} \cos (2 t)\right] / t$.

entanglement entropy of two free-fermionic chains connected by a narrow "transition" region is studied. The two chains are completely full or empty, respectively, while a density variation in the transition region is induced by a linear chemical potential. Interestingly, the von Neumann entropy of a block that includes the transition region grows logarithmically with the block size, with a prefactor 1/4, similar to Eq. (13).

It is useful to rewrite Eq. (12) as

$$
S_{\text {ansatz }}=\frac{\nu}{6} \log \left|y^{\frac{1}{2}} \sin (\pi y)\right|+\frac{\nu}{4} \log \left(L_{A}\right)+k_{\nu}^{\prime},
$$

with $y$ being the rescaled time as in Eq. (6). Clearly, the shifted entanglement $S_{A}(t)-\nu / 4 \log \left(L_{A}\right)$ is a function of only $y$.

The validity of Eq. (12) is further corroborated by comparing with the data shown in Fig. 4: dashed lines in the two panels (a) and (b) are one-parameter fits to Eq. (12), with $k_{\nu}^{\prime}$ the only fitting parameter, which are in perfect agreement with the numerical results. In order to demonstrate the scaling behavior Eq. (14) we plot $S_{A}(t)-\nu / 4 \log L_{A}$ versus $\nu v_{s} t /\left(2 L_{A}\right)$ in the insets of Fig. 4. All data for different sizes collapse on the same curve, further confirming Eq. (12).

Finite-size deviations from Eq. (12) are illustrated in Fig. 5, plotting $S_{A}(t)-S_{\text {ansatz }}(t)$ versus $\nu v_{s} t /\left(2 L_{A}\right)$ for $L_{A}=$ 50,100 (same data as in Fig. 4). The constant $k_{\nu}^{\prime}$ (cf. Eq. (12)) has been fitted and subtracted from the data. Finite-size corrections oscillate with time and vanish in the limit of large 
chains. We numerically checked that the formula

$$
S_{A}(t)-S_{\text {ansatz }}(t)=\frac{1}{t}\left(a_{1} \cos (t)+a_{2} \cos (2 t)\right)
$$

accurately describes the corrections at the intermediate time scales $0 \ll t \ll L_{A}$, as shown in the inset in Fig. 5. Symbols are data for $L_{A}=100$, while the continuous line is a fit to Eq. (15), with $a_{1}, a_{2}$ the fitting parameters. Notice the increasing behavior at $t \sim \nu L_{A} / v_{s}$, which could suggest a logarithmic correction as $\log (t) / t$. Similar corrections have been observed in the variance of the spin current after the quench from the "domain wall" state $|\cdots \uparrow \uparrow \uparrow \downarrow \downarrow \downarrow \cdots\rangle$ [102].

\section{ENTANGLEMENT PROPERTIES IN THE STEADY STATE}

This section is devoted to studying entanglement properties in the steady state after a generic geometric quench with arbitrary aspect ratio $\omega$ (cf. Fig. 1). This corresponds to time scales $t \gg t_{\ell}^{*}$ (cf. Fig. 3 (c)). Here we restrict our analysis to the $X X$ chain with periodic boundary conditions.

We first focus on the nature of the steady state after the quench. We show that it is not a thermal state, yet it reflects the initial half-filled Fermi sea in part $A$ of the chain. This observation allows us to derive an approximate analytic expression for the steady-state entanglement entropy, which is then checked against exact numerical results, finding good agreement, at least in the limit $L_{A} \ll L$. Remarkably, despite the non-thermal nature of the state, its entanglement is extensive. This is also confirmed through direct inspection of the so-called single-particle entanglement spectrum (ES).

\section{A. Non-thermal steady state}

After a Jordan-Wigner transformation (cf. Appendix A) the $X X$ Hamiltonian, obtained from Eq. (2) imposing $\Delta=0$, is recast in a free-fermionic form as

$$
\mathcal{H}_{X X}=-\frac{1}{2} \sum_{i=1}^{L-1}\left(c_{i}^{\dagger} c_{i+1}+c_{i} c_{i+1}^{\dagger}\right)
$$

with $c_{i}$ standard fermionic operators. Entanglement properties in free-fermionic models (cf. Appendix A) are fully characterized by the two-point correlation function $\mathbb{G}_{m, n} \equiv\left\langle c_{m}^{\dagger} c_{n}\right\rangle$ restricted to the subsystem, i.e., $m, n \in A[101,106-110]$. In the steady state, we numerically observe that the Fourier transform of $G_{m, n}, \widetilde{\mathbb{G}}_{k, k^{\prime}}$ is approximately diagonal, i.e., $\widetilde{\mathbb{G}}_{k, k^{\prime}} \approx n_{k} \delta_{k, k^{\prime}}$, with $n_{k}$ the subsystem momentum distribution function

$$
n_{k} \equiv\left\langle c_{k}^{\dagger} c_{k}\right\rangle=\frac{1}{L_{A}} \sum_{m, n} e^{i k(m-n)}\left\langle c_{m}^{\dagger} c_{n}\right\rangle .
$$

Here $k \equiv 2 \pi s / L_{A}$, with $s=0,1, \ldots L_{A}-1$, is the singleparticle momentum, $\langle\cdot\rangle$ denotes the expectation value with the post-quench wavefunction at time $t$, and $m, n \in\left[1, L_{A}\right]$.
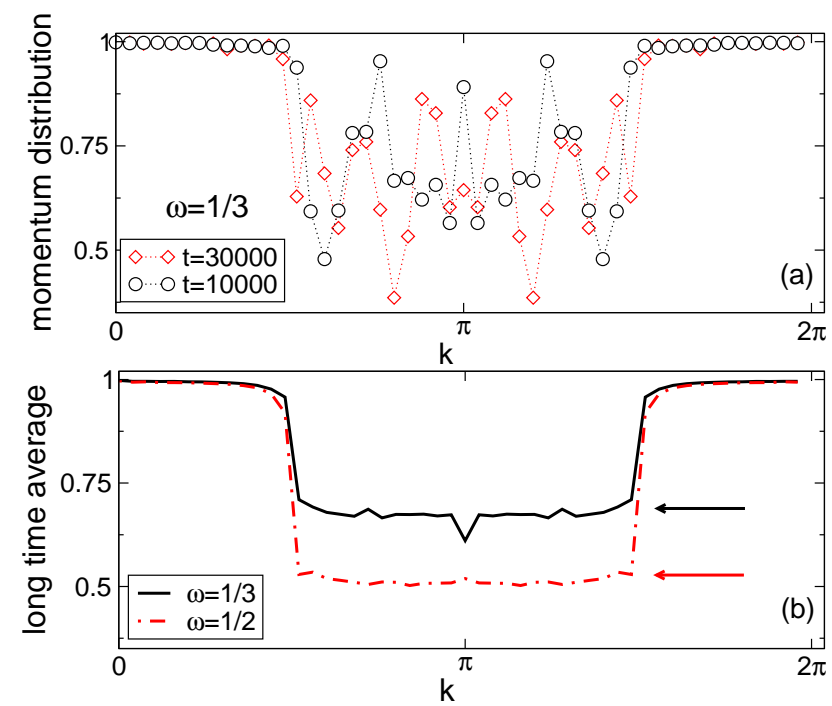

FIG. 6: Momentum distribution function $n_{k} \equiv\left\langle c_{k}^{\dagger} c_{k}\right\rangle$ restricted to part $A$ of the chain (cf. Fig. 1) after a geometric quench in the periodic $X X$ chain. Here $c_{k}, c_{k}^{\dagger}$ are the fermionic operators of the corresponding free-fermion chain (cf. Appendix A). (a) $n_{k}$ in the steady state $\left(t \gg t_{\ell}^{*}\right.$, cf. Fig. 3) plotted versus the single-particle momentum $0 \leq k \leq 2 \pi$. Data are for $L_{A}=50$. (b) Long-time average of $n_{k}$ (over the interval $20000 \leq t \leq 40000$ ). Full and dashed-dotted lines are for the geometric quench with aspect ratios $\omega=1 / 3$ and $\omega=1 / 2$, respectively (Fig. 1 ). The plateaux in the central region $(\pi / 2 \leq k \leq 3 / 2 \pi)$ correspond to $n_{k}=1-\omega$ (arrows in the figure).

$n_{k}$ is shown versus $k$ in Fig. 6 (a). Data are for the $X X$ chain with $L_{A}=50$ and the geometric quench with aspect ratio $\omega=1 / 3$. We restrict ourselves to times such that $t \gg t_{\ell}^{*}$ (cf. Fig. 3). Interestingly, apart from oscillations, a step-like structure is visible, which reflects the $t=0$ half-filled Fermi sea in part $A$ of the chain. The step-like form of $n_{k}$ is better visible in Fig. 6 (b). The continuous and dashed-dotted lines denote the time-averaged $n_{k}$ (in the interval $20000 \leq t \leq$ 40000) for two different quenches with $\omega=1 / 3$ and $\omega=$ $1 / 2$.

The form of $n_{k}$ can be derived in a semiclassical framework. At $t=0$ one can consider the excitations (particles) of the two independent chains $A$ and $B$ as uniformly distributed in each chain. Similarly, at $t \rightarrow \infty$ these are uniformly distributed in the final chain. Since the model is non-interacting, each mode preserves its momentum during the post-quench dynamics. The asymptotic (i.e., at $t \rightarrow \infty) n_{k}$ is then obtained as the "average" of the two initial Fermi seas $n_{k}^{0}(A)$ and $n_{k}^{0}(B)$ of parts $A$ and $B$, respectively. The result reads

$$
n_{k}=\omega n_{k}^{0}(A)+(1-\omega) n_{k}^{0}(B)
$$

where $\omega=L_{A} / L$ and $1-\omega=L_{B} / L_{A}$ have to be interpreted as the probabilities that a mode (with given momentum) is occupied by a particle originally in $A$ and $B$, respectively. Notice that in the reasoning above we are considering $L, L_{A} \rightarrow \infty$, i.e., we neglect the finite lattice spacing. Using that $n_{k}^{0}(A)$ and $n_{k}^{0}(B)$ are the half-filled and the filled Fermi 


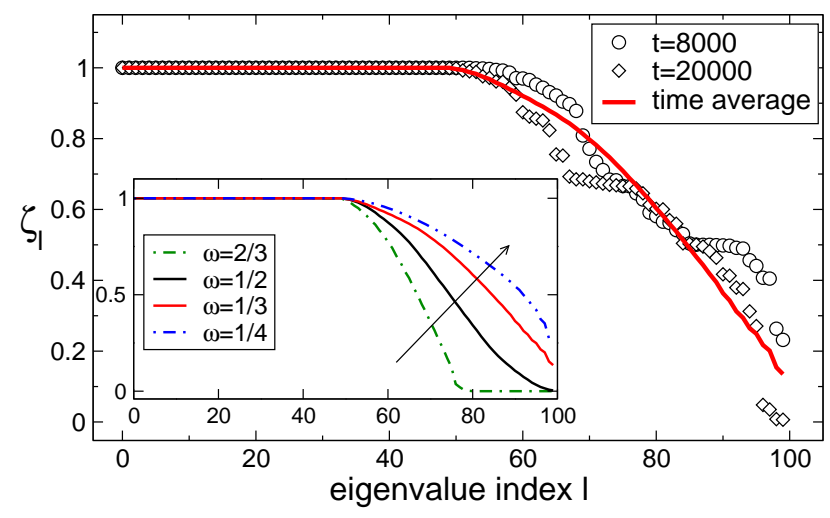

FIG. 7: Entanglement in the steady state after a geometric quench with $\omega=1 / 3$ in the periodic $X X$ chain. Single-particle entanglement spectrum levels $\zeta_{l}\left(l=1,2, \ldots, L_{A}\right)$ at long times $t>t_{\ell}^{*}$ (see Fig. 3). Data are exact results for a chain with $L_{A}=100$ and several times $8000 \leq t \leq 20000$. Note the first $L_{A} / 2$ levels with $\zeta_{l}=1$. The full line is the long time average. Inset: Long-time average of the single-particle entanglement spectrum for quenches with several values of $\omega \equiv L_{A} / L=2 / 3,1 / 2,1 / 3,1 / 4$. The arrow indicates decreasing $\omega$. Notice that the time averaged $\zeta_{l}$ are in general different from the time averaged $n_{k}$ in Fig. 6 (b).

seas, respectively, one obtains

$$
n_{k}=1-\omega \theta\left[k-\frac{\pi}{2}\right] \theta\left[\frac{3}{2} \pi-k\right],
$$

in perfect agreement with the numerical data in Figs. 6 (a) and (b). We should mention that similar results have been obtained studying the dynamics from the initial state $|m\rangle \otimes|-m\rangle$ in Ref. 33.

\section{B. Steady-state entanglement}

The entanglement entropy of subsystem $A$ at any time can be given as (cf. Appendix B)

$$
S_{A}(t)=-\sum_{l}\left[\zeta_{l} \log \zeta_{l}+\left(1-\zeta_{l}\right) \log \left(1-\zeta_{l}\right)\right]
$$

where $\zeta_{l}$, which are related to the single-particle entanglement spectrum (ES) (cf. Appendix B), are the eigenvalues of the two-point correlation matrix $\mathbb{G}_{m, n}$ restricted to part $A$ of the chain.

The behavior of $\zeta_{l}$ in the steady state is illustrated in Fig. 7 for the geometric quench with $\omega=L_{A} / L=1 / 3$ and fixed $L_{A}=100$. The continuous line is the (long) time average of the levels. At time $t>t_{\ell}^{*}$ there are $L_{A} / 2$ (i.e., half of the levels) with $\zeta_{l}=1$, which do not contribute to the entropy (cf. Eq. (20)). The remaining $L_{A} / 2$ are distributed over the whole interval $(0,1)$. The existence of an extensive number of levels with $\zeta_{l} \sim 1 / 2$ suggests that $S_{A}(t)$ is extensive in the steady state. This is dramatically different in the equilibrium ground state, where only few levels of the single-particle entanglement spectrum contribute in Eq. (20) (cf. Fig. 13 in Appendix B).

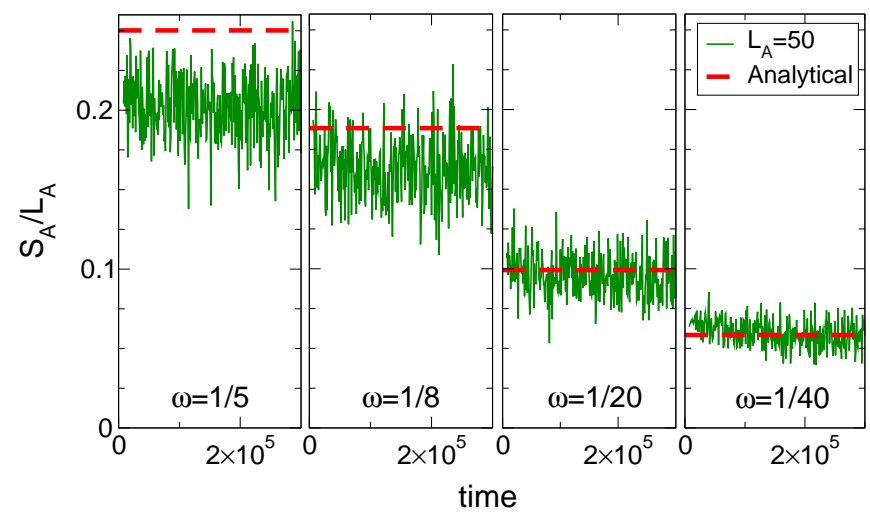

FIG. 8: Extensive entanglement in the steady state after a geometric quench. Data are for the periodic $X X$ chain. von Neumann entropy $S_{A}$ for part $A$ of the chain (cf. Fig. 1) at large times ( $t \gg t_{\ell}^{*}$, see Fig. 3): $S_{A} / L_{A}$ plotted versus time $t$ for quenches with several aspect ratios $\omega=L_{A} / L=1 / 5,1 / 8,1 / 20,1 / 40$ and $L_{A}=50$ (the same scale is used on both axis in all the panels). The dashed line is $S_{A} / L_{A}=-[\omega \log \omega+(1-\omega) \log (1-\omega)] / 2$.

The behavior of $\zeta_{l}$ upon varying the aspect ratio $\omega$ is illustrated in the inset in Fig. 7, showing the time-averaged levels for $\omega=2 / 3,1 / 2,1 / 3,1 / 4$. Irrespective of $\omega$ an extensive fraction $\left(\sim L_{A} / 2\right)$ of levels is in the region $\zeta_{l} \approx 1$. Moreover, as $\omega$ decreases the whole distribution is shifted towards $\zeta_{l}=1$, signaling that, although extensive behavior persists at any $\omega$, the actual value of the entropy decreases as $\omega \rightarrow 0$.

Finally, the scenario outlined above can be justified using Eq. (19), i.e., neglecting the oscillations in Fig. 6 (a). Within this approximation, $\mathbb{G}_{m, n}$ has $L_{A} / 2$ identical eigenvalues $\zeta_{l}=1-\omega$ (and $L_{A} / 2$ unit eigenvalues). Notice that these are different from the long time average of $\zeta_{l}$ in Fig. 7, suggesting that the diagonal approximation $\widetilde{\mathbb{G}}_{k, k^{\prime}} \approx n_{k} \delta_{k, k^{\prime}}$ might be too crude. The von Neumann entropy, using Eq. (20) is then

$$
\frac{S_{A}}{L_{A}}=-\frac{1}{2}[\omega \log \omega+(1-\omega) \log (1-\omega)] .
$$

The comparison between Eq. (21) and the exact data is shown in Fig. 8, focusing on the geometric quenches with $\omega=$ $1 / 5,1 / 8,1 / 20,1 / 40$ (panels from left to right in the figure). The continuous lines are data for $S_{A} / L_{A}$ at fixed $L_{A}=50$ and $10^{4}<t<10^{6}$. Clearly, $S_{A} / L_{A} \rightarrow 0$ upon decreasing $\omega$, as expected (cf. inset in Fig. 7). In each panel, the value according to Eq. (21) is shown as a dashed line. At $\omega=1 / 5$ and $\omega=1 / 8$ some deviations from Eq. (21) are observed, which have to be interpreted as finite-size effects, due to the fact that $L_{A} \sim L$. In the limit $L_{A} \ll L$ (equivalent to $\omega \rightarrow 0$ ), Eq. (21) is in remarkably good agreement with the exact data.

It is interesting to investigate the entanglement fluctuations in the steady state. These are illustrated in Fig. 9 plotting the rescaled von Neumann entropy $S_{A} / L_{A}$ versus $t / L_{A}^{2}$. Data are for the open $X X$ chain with $L=100,200,400$ and a geometric quench with aspect ratio $\omega=1 / 2$ (cf. Fig. 1). Here we focus on intermediate and long time scales after the quench, i.e., $t \gg t_{s}^{*}$ (see Fig. 3). Remarkably, in Fig. 9 all the data 


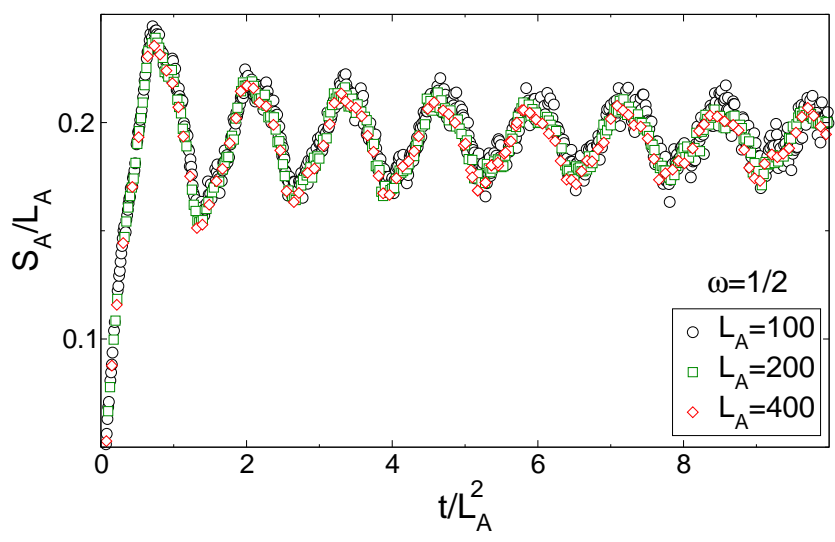

FIG. 9: Fluctuations of the entanglement entropy in the steady state after a geometric quench with aspect ratio $\omega \equiv L_{A} / L=1 / 2$. Data are for the open $X X$ chain with $L=100,200,400$ : rescaled von Neumann entropy $S_{A} / L_{A}$ plotted versus the rescaled time $t / L_{A}^{2}$. Notice that partial revivals (oscillations) persist in the long time regime, and do not decay with the chain size.

collapse on the same curve, confirming that the steady state exhibits extensive entanglement, in agreeement with the semiclassical result Eq. (21). However, oscillating deviations from the steady state value are observed, with period $\sim 1 / L_{A}^{2}$ and amplitude $\sim L_{A}$. Notice that at fixed $t / L_{A}^{2}$ these oscillations do not vanish in the limit $L_{A} \rightarrow \infty$.

\section{ENTANGLEMENT RELAXATION AFTER THE INFINITE CHAIN QUENCH}

In this section we discuss the real-time entanglement dynamics after the infinite-chain geometric quench, which corresponds to the limit $\omega \rightarrow 0$, at fixed finite $L_{A}$ (see Fig. 1). Here we focus on time scales $t>t_{s}^{*}$ (for $t<t_{s}^{*}$, one has the same behavior as in Sec. V), considering the $X X$ chain with periodic boundary conditions. Although we are interested in the limit $\omega \rightarrow 0$, in practice we consider finite (large) $\omega$ restricting ourselves only to $t<L-L_{A}=L_{A}\left(\omega^{-1}-1\right)$, to avoid reflections at the boundaries of the chain.

First, one has $S_{A}(t) \rightarrow 0$ at $t \rightarrow \infty$, since the wavefunction becomes a product state in the limit $\omega \rightarrow 0$. However, the entanglement relaxation dynamics, at any time $t>t_{s}^{*}$, is described by a scaling function $f_{\ell}(z)$ of the rescaled time $z=t / L_{A}^{2}$. Additionally, two different dynamical regimes appear: while $f_{\ell}(z) \sim-\log (z)$ at $z \lesssim 1$, a crossover to $f_{\ell}(z) \sim 1 / z+1 / z \log (z)$ occurs around $z \sim 1$. The latter behavior can be calculated analytically.

All these features are present in the data shown in Fig. 10, plotting $S_{A}(t)$ versus $z \equiv t / L_{A}^{2}$ for a geometric quench with $\omega=1 / 30$ and several $L_{A}$. The perfect data collapse provides robust evidence that the entanglement dynamics at $t>t_{s}^{*}$ is described by a scaling function $f_{\ell}(z)$. The behavior of $f_{\ell}(z)$ at $z \rightarrow \infty$ is given analytically as (cf. Appendix E)

$$
S_{A}(t) \approx \frac{L_{A}^{2}}{2 \pi t}\left[1-\log \frac{L_{A}^{2}}{2 \pi t}\right] .
$$

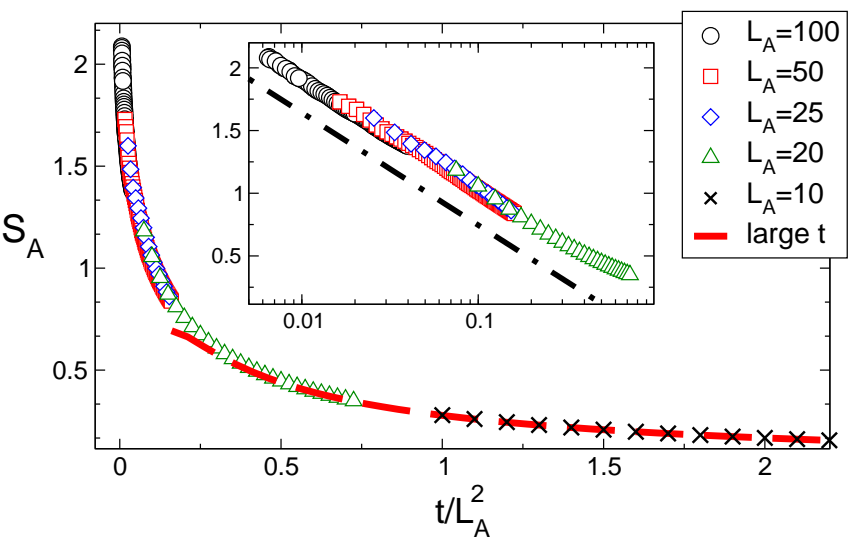

FIG. 10: Entanglement relaxation after the geometric quench with aspect ratio $\omega \equiv L_{A} / L \ll 1$ in the periodic $X X$ chain. Symbols are exact numerical data for $L_{A}=10,20,25,50,100$. Rescaled entropy dynamics: $S_{A}$ versus $t / L_{A}^{2}$. Perfect data collapse is observed for all $L_{A}$ and times. The dashed line is the analytic result at $t \rightarrow \infty$. Inset: same as in the main figure at $t / L_{A}^{2}<1$. Note the logarithmic scale on the $x$-axis. The dashed dotted line is $-0.4 \log \left(t / L_{A}^{2}\right)$.

This is shown in Fig. 10 as a dashed line, in perfect agreement with the numerical data already at $t / L_{A}^{2} \sim 1 / 2$. On the other hand, at $t / L_{A}^{2} \ll 1$, one has the strikingly different behavior $S_{A}(t)=-\alpha \log \left(t / L_{A}^{2}\right)+\beta$, as numerically demonstrated in the inset of Fig. 10. In particular, a fit of the numerical data gives $\alpha \approx 0.4$ (dashed-dotted line in the figure).

\section{GEOMETRIC QUENCH IN THE $X X Z$ CHAIN}

We now turn to the post-quench dynamics in interacting models, considering the $X X Z$ chain in the gapless phase at $-1<\Delta \leq 1$. We restrict ourselves to short time scales, which can be accessed efficiently using tDMRG [77, 78, 93, 94].

We provide numerical evidence that qualitative and quantitative features are similar to the $X X$ chain. First, after the quench a magnetization wavefront forms, spreading ballistically in the two parts of the chain $A$ and $B$ (cf. Fig. 1). However, while in the $X X$ chain the two wavefront edges propagate with the same velocity (i.e., $v=1$, see Sec. III), here two different velocities appear. More precisely, the propagation in parts $A$ and $B$ happens at the spinon velocity $v_{s}(\Delta)$ and $v=1$, respectively. Interestingly, we find that the central region of the wavefront is described by Eq. (10).

On the other hand, the entanglement dynamics is well described by the same formula derived for the $X X$ chain (cf. Eq. (12)). However, as the wavefronts (and consequently the information) propagation is anisotropic in the two parts of the chain, a remarkable difference is that one has to replace $v_{s} \rightarrow v_{e}$ in Eq. (12), with $v_{e}$ being an effective entanglement spreading rate. We numerically find that $v_{e} \approx v_{s}$ for $\Delta<0$ (i.e., the entanglement spreads with the wavefront edge velocity), whereas one has $v_{e}<v_{s}$ at $\Delta>0$. 

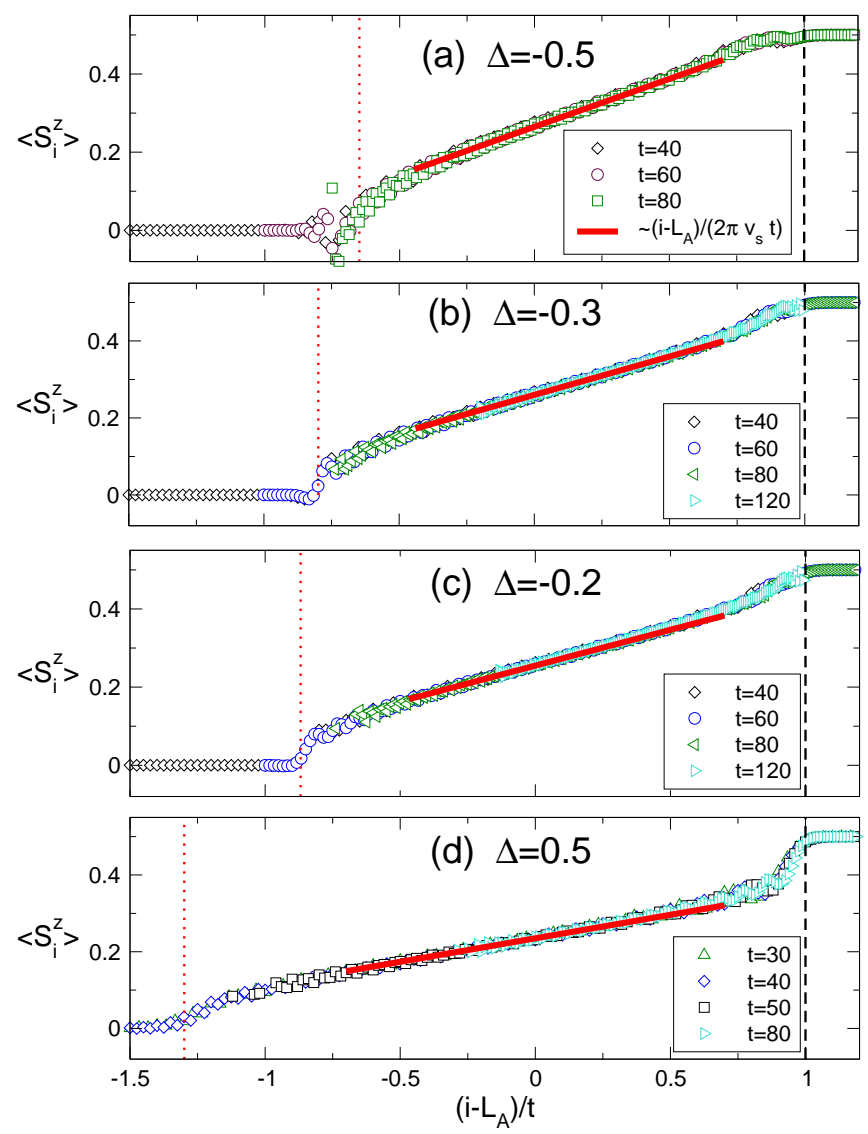

FIG. 11: Magnetization wavefront after a geometric quench with aspect ratio $\omega \equiv L_{A} / L=1 / 3$ in the $X X Z$ spin chain. Symbols are tDMRG data for $L_{A}=60$ and anisotropies $\Delta=$ $-0.5,-0.3,-0.2,0.5$ (panels (a)-(d) in the figure). The same scale is used on the $x$-axis in all panels. The local magnetization $\left\langle S_{i}^{z}\right\rangle$ is plotted versus $\left(i-L_{A}\right) / t$, with $i$ being the position in the chain. All the data collapse on the same $\Delta$ dependent scaling function. The dashed vertical line corresponds to $\left(i-L_{A}\right) / t=1$, while the dasheddotted one is $\left(i-L_{A}\right) / t=-v_{s}(\Delta)$, with $v_{s}(\Delta)$ the spinon velocity. The continuous lines are fits to $s_{0}+\left(i-L_{A}\right) /\left(2 \pi v_{s}(\Delta) t\right)$, with $s_{0} \approx 1 / 4$ the fitting parameter.

\section{A. Ballistic wavefront propagation}

The local magnetization $\left\langle S_{i}^{z}(t)\right\rangle$ as a function of the site position $i$ in the chain is shown in Fig. 11 for several times after the geometric quench. We restrict ourselves to $\omega=1 / 3$, showing data at fixed $L_{A}=60$ and several values of the anisotropy $\Delta$ in the spin-liquid phase $(\Delta=$ $-0.5,-0.3,-0.2,0.5$, panels (a)-(d) in the figure). Symbols denote tDMRG data for an $X X Z$ chain with open boundary conditions and $t \ll L_{A}$ to avoid effects from reflections at the boundary of the chain.

The formation of two propagating wavefronts at $t>0$ is clearly visible for all values of $\Delta$. Their left and right edges propagate ballistically in the two parts of the chain (see also Ref. 79, 111). This is illustrated plotting $\left\langle S_{i}^{z}\right\rangle$ versus the rescaled variable $\left(i-L_{A}\right) / t$. At each $\Delta$, the data collapse on the same function for all times and positions.
The vertical dashed-dotted line in Fig. 11 marks the point $\left(i-L_{A}\right) / t=-v_{s}$. For the $X X Z$ chain in the zero magnetization sector $v_{s}$ is given as [112]

$$
v_{s}(\Delta)=\frac{\pi}{2} \frac{\sin \gamma}{\gamma} \text { with } \quad \cos \gamma=\Delta .
$$

Clearly, $\left\langle S_{i}^{z}\right\rangle=0$ at $\left(i-L_{A}\right) / t=-v_{s}$, demonstrating that the left edge of the wavefront propagates with $v_{s}$. On the other hand, the right one propagates at unit velocity (the dashed lines in all panels mark the point $\left.\left(i-L_{A}\right) / t=1\right)$. Although the full scaling function $\left\langle S_{i}^{z}\right\rangle \equiv m\left(\left(i-L_{A}\right) / t\right)$ is not easily accessible, at $\left(i-L_{A}\right) \ll t$ (central region in the panels in Fig. 11) the magnetization profile exhibits the linear behavior $\left\langle S_{i}^{z}\right\rangle \sim\left(i-L_{A}\right) / t$. It is reasonable that this is given analytically by (as a generalization of Eq. (10))

$$
\left\langle S_{i}^{z}\right\rangle \approx s_{0}+\frac{i-L_{A}}{2 \pi v_{s}(\Delta) t}
$$

The validity of Eq. (24) is confirmed in Fig. 11. Continuous lines in the figure are fits to Eq. (24) (with $s_{0} \approx 1 / 4$ the fitting parameter), and are in excellent agreement with the tDMRG data.

\section{B. Short-time entanglement dynamics}

In this section we investigate the entanglement spreading after a geometric quench in the (open) $X X Z$ chain in the gapless phase (i.e., $-1<\Delta \leq 1$ ). At short time scales that can be accessed by tDMRG it is natural to generalize the result at $\Delta=0$ (cf. Eq. (12)) as

$$
S_{\text {ansatz }}(t)=\frac{\nu}{6} \log \left[L_{A}^{\frac{3}{2}}\left(\frac{\nu v_{e} t}{2 L_{A}}\right)^{\frac{1}{2}} \sin \frac{\nu \pi v_{e} t}{2 L_{A}}\right]+k_{\nu}^{\prime}
$$

with $\nu=1$ for open and $\nu=2$ for periodic boundary conditions, $k_{\nu}^{\prime}$ a $\Delta$-dependent constant, and $v_{e}$ an entanglement spreading rate. Equation (25) is expected to hold at $t \ll 2 L_{B}$, although we are not able to provide its precise regime of validity, which would require the exact expression for $v_{e}$. From Eq. (25) one finds that $S_{A}(t)-\nu / 4 \log \left(L_{A}\right)$ is a scaling function of $t / L_{A}$. The validity of Eq. (25) is shown in Fig. 12, considering tDMRG data for the $X X Z$ chain at $\Delta=-1 / 2$ (panel (a) in the figure) and $\Delta=1 / 2$ (panel (b)). We provide data for $L_{A}=20,30,40,60$, restricting ourselves to a geometric quench with $\omega=1 / 3$. Strikingly, all the data for different system sizes collapse on the same scaling curve, in agreement with Eq. (25). To further proceed we fit the data to Eq. (25) ( $k_{\nu}^{\prime}$ and $v_{e}$ being the only fitting parameters). Remarkably, at $\Delta=-1 / 2$, we obtain $v_{e} \approx v_{s}$ (the vertical dotted line in Fig. 12 marks the point at $2 L_{A} / t=v_{s}$ ). Also, we numerically verified that $v_{e} \approx v_{s}$ in the whole interval $-1<\Delta \leq 0$. However, at $\Delta=1 / 2$ we obtain $v_{e} \approx 1.13<v_{s} \approx 1.3$ (the vertical-dashed line in Fig. 12 marks the point $\left.t /\left(2 L_{A}\right)=1 / v_{s}\right)$. Our analysis suggests that although the information spreading between the two parts $A$ and $B$ of the chain is associated with the wavefront propagation, the spreading rate $v_{e}$ is not a trivial function of the wavefront edges' velocities. 

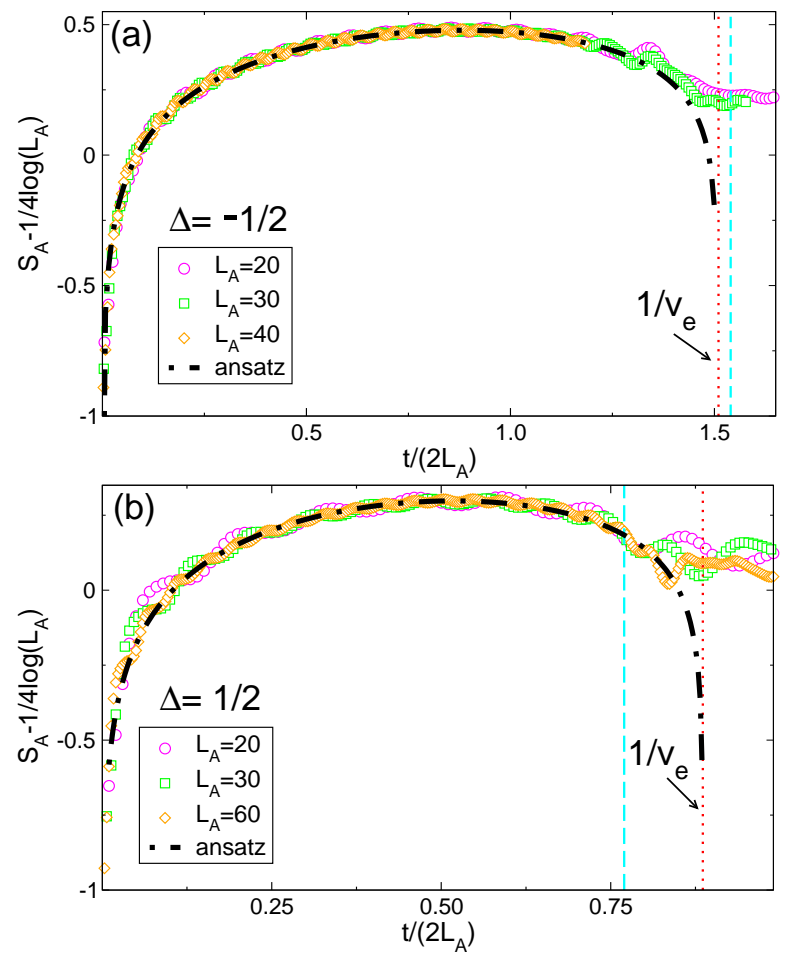

FIG. 12: Entanglement spreading after the geometric quench with aspect ratio $\omega \equiv L_{A} / L=1 / 3$ (see Fig. 1) in the $X X Z$ spin chain at $\Delta=-1 / 2$ (a) and $\Delta=1 / 2$ (b). (a) Shifted von Neumann entropy $S_{A}-1 / 4 \log L_{A}$ versus $t /\left(2 L_{A}\right)$. Symbols are tDMRG data for $L_{A}=20,30,40$. Notice the perfect data collapse for all sizes and times. The dashed line are fits to $S_{\text {ansatz }}$ (Eq. (25)), with $v_{e}$ (entanglement spreading rate) and $k_{\nu}^{\prime}$ the fitting parameters. The vertical dotted line marks the point $2 L_{A} / t=v_{e}$. The fit gives $v_{e} \approx$ $v_{s}(\Delta)$. (b) Same as in (a) for $\Delta=1 / 2$ and $L_{A}=20,30,60$. The fit to Eq. (25) now yields $v_{e} \approx 1.13<v_{s} \approx 1.3$. In both panels, the vertical dashed line is $1 / v_{s}(\Delta)$.

\section{SUMMARY AND CONCLUSIONS}

In this work we investigated the entanglement dynamics after a geometric quench in the $X X Z$ chain in the gapless phase, both analytically and numerically. The initial state after the quench is obtained joining two chains $A$ and $B$, of lengths $L_{A}$ and $L_{B}$, prepared in the ground state of the $X X Z$ chain in the sector with zero and maximum magnetization, respectively. The latter is the fully polarized state, which can be a high-energy eigenstate of the model, depending on the exchange anisotropy. Equivalently, in the language of interacting fermions confined in a hard-wall trap, the geometric quench corresponds to a sudden change in the trap size. From the energy point of view, this quench falls into the class of global quenches, since the excess energy density above the ground state of the final Hamiltonian is finite. On the other hand, both $A$ and $B$ are in eigenstates of the $X X Z$ model, implying that the post-quench dynamics originates locally from a "defect" at the interface between the two chains, as in typical local quenches.

The entanglement growth after the quench is associated with the formation of a magnetization wavefront, whose edges propagate ballistically in the two parts of the chain, at different velocities. To be precise, while the wavefront expands in part $A$ with the spinon velocity, in part $B$ this happens at unit velocity. For the $X X$ chain we derived the exact analytical expression of the wavefront profile using free-fermionic techniques and semiclassical arguments. For the $X X Z$ model we found that the central region of the wavefront is described by a simple function, which depends on the spinon velocity.

Focusing on the $X X$ chain we observed that the entanglement dynamics after the quench exhibits several interesting dynamical regimes. Specifically, at $t \leq t_{s}^{*} \sim L_{A} / v_{s}$ (short time scales) the von Neumann entropy increases logarithmically. Moreover, while the well-known CFT result [21-23] for the entanglement growth in local quenches does not apply, we provided an analytic formula, derived from heuristic arguments, that describes accurately the short-time entanglement dynamics. Remarkably, the entanglement entropy exhibits the scaling behavior $S_{A}(t)=f_{s}(y)+s\left(L_{A}\right)$, with $y=t / L_{A}$, $s\left(L_{A}\right)=\nu / 4 \log \left(L_{A}\right)$ (here $\nu=1,2$ for open and periodic boundary conditions, respectively), and $f_{s}(y)$ a scaling function. At larger times $\left(t_{s}^{*} \leq t \leq t_{\ell}^{*}\right.$, with $\left.t_{\ell}^{*} \sim L_{A}^{2} / v_{s}\right)$ the von Neumann entropy shows partial revivals of the short-time dynamics superposed with a power-law increase $S_{A} \sim t^{\alpha}$. We numerically found $\alpha<1$.

At very long times $t \geq t_{\ell}^{*}$ the system reaches a steady state and the entanglement entropy saturates, apart from oscillations. As expected, since the model is integrable, the steady state is not thermal. More precisely, we observed that the subsystem momentum distribution function shows discontinuities at $k=\pi / 2$, reflecting the initial half-filled Fermi sea in part $A$ of the chain. Finally, we provided numerical and analytical evidence that the steady-state entanglement is extensive.

We also considered the geometric quench from a finite to the infinite chain. While at large times one has $S_{A} \rightarrow 0$, reflecting the wavefunction being an almost perfect product state, the entanglement relaxation dynamics exhibits the scaling form $S_{A}(t)=f_{\ell}(z)$, with $z \equiv t / L_{A}^{2}$. Interestingly, the behavior of $f_{\ell}(z)$ changes dramatically at $z \sim 1$ (i.e., $\left.t \sim L_{A}^{2}\right)$. Namely, we numerically observed that $f_{\ell}(z) \approx$ $-\gamma \log (z)$, with $\gamma \approx 0.4$ at $z \lesssim 1$. On the other hand, at $z \gtrsim 1$ we derived analytically $f_{\ell}(z) \approx 1 / z(1-\log (1 / z))$.

Finally, by means of tDMRG simulations we discussed the role of interactions in the short-time entanglement dynamics, considering the $X X Z$ chain in the gapless phase $-1<\Delta \leq 1$. Interestingly, we numerically demonstrated that the same formula conjectured for the free-fermion case fully reproduces the short-time entanglement dynamics after the quench. Due to the anisotropic propagation of the wavefront in the two parts of the chain $A$ and $B$, the entanglement spreading rate is not trivially related to the velocity of a single wavefront edge.

\section{ACKNOWLEDGEMENTS}

We acknowledge very fruitful discussions with J.-S. Caux, P. Calabrese, M. Fagotti, M. Haque, J. Mossel, G. Palacios, 
P. Ribeiro. V.A. thanks J.-S. Caux, J. Mossel, and G. Palacios for valuable discussions in a related project, from which this work originated. We thank F. Iglói and J. H. H. Perk for useful comments on a previous version of the manuscript.

\section{Appendix A: Diagonalization of the spin- $1 / 2 X X$ chain}

The spin- $1 / 2$ open $X X$ chain [113-117] of length $L$ in an external magnetic field $h$ is defined as

$$
\mathcal{H}_{X X}=-J \sum_{i=1}^{L-1}\left(S_{i}^{x} S_{i+1}^{x}+S_{i}^{y} S_{i+1}^{y}\right)+h \sum_{i=1}^{L} S_{i}^{z},
$$

with $S_{i}^{x, y, z} \equiv \sigma_{i}^{x, y, z} / 2, \sigma_{i}^{\alpha}$ being the Pauli matrices acting on site $i$. For periodic boundary conditions one has an extra term in Eq. (A1) connecting site $L$ with site 1 . Hereafter we fix $J=$ 1 for convenience. After the Jordan-Wigner transformation

$$
c_{i}=\left(\prod_{m=1}^{i-1} \sigma_{m}^{z}\right) \frac{\sigma_{i}^{x}-i \sigma_{i}^{y}}{2},
$$

Eq. (A1) is recast in the free-fermionic form

$$
\mathcal{H}_{X X}=-\frac{1}{2} \sum_{i=1}^{L-1}\left(c_{i}^{\dagger} c_{i+1}+c_{i} c_{i+1}^{\dagger}\right)+\frac{h}{2} \sum_{i=1}^{L-1} c_{i}^{\dagger} c_{i},
$$

with $c_{i}$ spinless fermionic operators satisfying the canonical anticommutation relations $\left\{c_{m}, c_{n}^{\dagger}\right\}=\delta_{m, n}$. Notice in Eq. (A2) the non local term (Jordan-Wigner string) in the brackets. The mapping between Eq. (A1) and Eq. (A3) is exact apart from boundary terms (that we neglect here) giving a vanishing contribution (as $1 / L$ ) to physical quantities in the large chain limit.

Periodic boundary conditions $(p b c)$.- For periodic boundary conditions the spectrum of Eq. (A3) can be obtained going to momentum space. After defining the Fourier transformed operators $c_{k}$ as

$$
c_{k}=\frac{1}{\sqrt{L}} \sum_{m=1}^{L} e^{i \frac{2 \pi k}{L} m} c_{m}
$$

and substituting in Eq. (A3), one obtains the single-particle dispersion $E_{k}$ of the $X X$ chain as

$$
E_{k}=-\cos \frac{2 \pi k}{L}+h \quad \text { with } \quad k=0,1, \ldots, L-1,
$$

with $2 \pi k / L$ and $E_{k}$ the single-particle momenta and energies, respectively. The single-particle eigenstate $\left|v_{k}\right\rangle$ corresponding to the eigenvalue $E_{k}$ reads

$$
\left|v_{k}\right\rangle=\frac{1}{\sqrt{L}} \sum_{m=1}^{L} e^{i \frac{2 \pi k}{L} m} c_{m}^{\dagger}|0\rangle,
$$

with $|0\rangle$ denoting the vacuum state for the fermions.
Open boundary conditions $(o b c)$.- For the $X X$ chain with open boundary conditions one has instead

$$
E_{k}^{\prime}=-\cos \frac{\pi k}{L+1}+h \quad \text { with } \quad k=0,1, \ldots, L-1,
$$

while the single-particle eigenstates are

$$
\left|v_{k}^{\prime}\right\rangle=\sqrt{\frac{2}{L+1}} \sum_{m=1}^{L} \sin \left[\frac{\pi m k}{L+1}\right] c_{m}^{\dagger}|0\rangle .
$$

The spectrum of the model (cf. Eq. (A5) and Eq. (A7)) is gapless in the thermodynamic limit at $|h|<1$, while it is gapped otherwise. The ground state at $h=1(h=-1)$ corresponds to an empty (fully filled) band.

The ground state of the $X X$ chain is obtained by filling the single-particle levels (cf. Eqs. (A5) and (A7)) below the Fermi level $k_{F}=L /(2 \pi) \cos ^{-1}(h)$ and $k_{F}=(L+1) / \pi \cos ^{-1}(h)$ for periodic and open boundary conditions, respectively. Notice that for convenience, the Fermi level $k_{F}$ is defined as an integer. In this work we restrict ourselves to the $X X$ chain with zero magnetic field $(h=0)$.

\section{Appendix B: Entanglement entropies in free-fermionic chains}

Here we briefly review how to calculate the entanglement entropy for a generic eigenstate of a free-fermionic model [101, 106-110, 118], focusing, in particular, on the ground-state entropy. The von Neumann entropy (and Renyi entropies as well) of a single interval $A \equiv\left[1, L_{A}\right]$ (of length $L_{A}$ ) embedded in a free-fermionic chain can be obtained from the two-point correlation function $\mathbb{G}_{m, n}$ restricted to the subsystem $A$

$$
\mathbb{G}_{m, n}=\left\langle c_{m}^{\dagger} c_{n}\right\rangle \quad \text { with } \quad m, n=1,2, \ldots L_{A} .
$$

Here $\langle\cdot\rangle$ denotes the expectation value over a generic eigenstate of Eq. (A3).

Ground-state correlation matrix.- The correlation matrix $\mathbb{G}_{m, n}$ (cf. Eq. (B1)) for a generic eigenstate of Eq. (A3) can be obtained using the explicit form of the single-particle eigenvectors Eq. (A6) and Eq. (A8). For the ground state of Eq. (A3) and periodic boundary conditions one obtains

$$
\mathbb{G}_{m, n}^{(\mathrm{pbc})}=\frac{2}{L} \sum_{k=0}^{L / 4-1} \cos \left[\frac{2 \pi(m-n) k}{L}\right]-\frac{1}{L} .
$$

Performing the summation over $k$ one obtains

$$
\mathbb{G}_{m, n}^{(\mathrm{pbc})}=\frac{1}{L} \frac{\sin \left[\left(\frac{\pi}{2}-\frac{\pi}{L}\right)(m-n)\right]}{\sin \left[\frac{\pi}{L}(m-n)\right]} .
$$

In the limit of an infinite chain, Eq. (B3) reduces to

$$
\mathbb{G}_{m, n}^{(\mathrm{pbc})}(L \gg 1) \rightarrow \frac{\sin \left[\frac{\pi}{2}(m-n)\right]}{\pi(m-n)} .
$$


Finally, for open boundary conditions $\mathbb{G}_{m, n}^{(\mathrm{obc})}$ reads

$$
\begin{array}{r}
\mathbb{G}_{m, n}^{(\mathrm{obc})}=\frac{1}{2(L+1)}\left[\frac{\sin \frac{\pi}{2}(m-n)}{\sin \frac{\pi}{2(L+1)}(m-n)}-\right. \\
\left.\frac{\sin \frac{\pi}{2}(m+n)}{\sin \frac{\pi}{2(L+1)}(m+n)}\right] .
\end{array}
$$

For free-fermionic models the reduced density matrix for $A$ can be written as

$$
\rho_{A}=\frac{1}{Z} \exp \left(-\mathcal{H}_{E}\right)
$$

where $Z$ ensures the normalization $\operatorname{Tr} \rho_{A}=1$, and $\mathcal{H}_{E}$ is the so-called entanglement Hamiltonian. The spectrum of the reduced density matrix $\rho_{A}$ in Eq. (B6), which is expressed in the free-fermionic variables $c_{i}$, coincides with that of the reduced density matrix of the same block $A$ expressed in the original spin variables $\sigma_{i}^{x, y, z}$. Since the Jordan-Wigner transformation Eq. (A2) is non-local, this is a non-trivial fact, and it rests on subsystem $A$ being a single interval. In fact, it does not remain true for two (or many) disjoint intervals [119-124].

The spectrum of $\mathcal{H}_{E}$ (single-particle entanglement spectrum) is of the free-type, reflecting the original Hamiltonian Eq. (A3) being quadratic, and its single-particle levels $\epsilon_{l}$ (l being an arbitrary label) are obtained from the eigenvalues $\zeta_{l}$ of $\mathbb{G}_{m, n}$ (cf. Eq. (B1)) as

$$
\epsilon_{l}=\log \left[\frac{1-\zeta_{l}}{\zeta_{l}}\right] .
$$

Finally, the von Neumann entropy $S_{A}$ of $A$ is obtained as

$$
S_{A}=\sum_{l=1}^{L_{A}}\left[\log \left(1+e^{-\epsilon_{l}}\right)+\frac{\epsilon_{l}}{1+e^{\epsilon_{l}}}\right] .
$$

Equivalently, in terms of $\zeta_{k}$ one can write

$$
S_{A}=\sum_{l=1}^{L_{A}}\left[\zeta_{l} \log \zeta_{l}-\left(1-\zeta_{l}\right) \log \left(1-\zeta_{l}\right)\right] .
$$

It is noteworthy that the term in the sum in Eq. (B9) has a maximum at $\zeta_{l}=1 / 2$, whereas it is vanishing for $\zeta_{l}=0,1$.

An example of a single-particle entanglement spectrum is shown in Fig. 13 plotting the eigenvalues $\zeta_{l}$ for a periodic $X X$ chain with $L=300$ and block $A$ with $L_{A}=75$ and $L_{A}=$ 150 (rhombi and circles in the figure, respectively). Clearly, a large fraction of the spectrum (levels with $\zeta_{l}=0,1$ ) does not contribute to the entanglement entropy (cf. Eq. (B9)).

\section{Appendix C: Entanglement entropies after a geometric quench}

In this section we illustrate the calculation of the entanglement entropy at any time after a generic (i.e., with arbitrary aspect ratio $\omega$, see Fig. 1) geometric quench in the $X X$ chain. Similar results can be obtained for the $1 D X Y$ model (see Refs. 114-117) or the transverse-field Ising chain (see

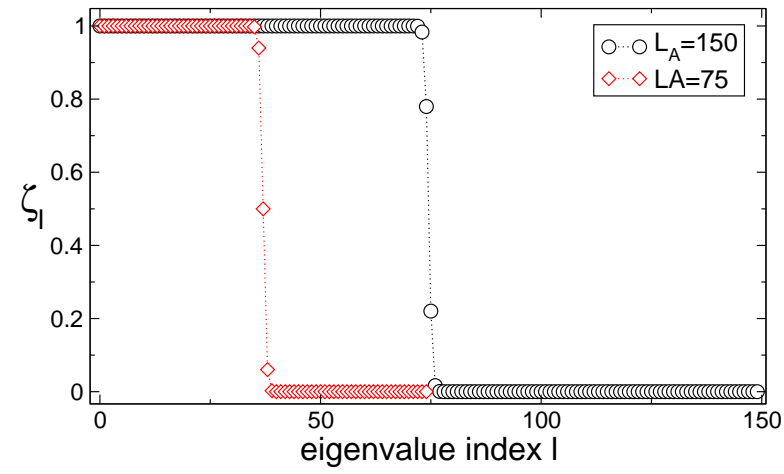

FIG. 13: Ground-state single-particle entanglement spectrum (ES) levels for the $X X$ chain. Symbols are exact results for a chain of length $L=300$. The ES levels are for a subsystem with $L_{A}=75$ (rhombi) and $L_{A}=150$ (circles). Only few ES levels (with $\zeta_{l} \neq$ $0,1)$ contribute to the entanglement entropy.

Refs 125, 126 and references therein). For simplicity, here we consider the situation in which both the initial chain $A$ (see Fig. 1) and the final one have periodic boundary conditions. Notice that this implies that the quench protocol (see Fig. 1) involves a "cut and glue" step.

At $t=0$ the initial state of the $X X$ chain is obtained by gluing together the zero-magnetization ground state of a chain of length $L_{A}$ with a fully-polarized state $|F\rangle \equiv|\uparrow \uparrow \uparrow \ldots \uparrow\rangle$ of length $L_{B} \equiv L-L_{A}$. The two-point correlation matrix $\mathbb{G}_{m, n}(t)$ at $t=0$ is given as

$$
\mathbb{G}_{m, n}(0)=\left\{\begin{array}{cc}
\mathbb{G}_{m, n}^{(\text {init })} & \text { if } \\
\delta_{m, n} & \text { otherwise }
\end{array}(m, n) \in A\right.
$$

with $\mathbb{G}_{m, n}^{\text {(init) }}$ the $t=0$ correlation function in part $A$ of the chain, and $\delta_{m, n}$ the Kronecker delta. Here we choose $\mathbb{G}_{m, n}^{(\text {init })}=$ $\mathbb{G}_{m, n}^{(\mathrm{pbc})}$ (Eq. (B3) after replacing $L \rightarrow L_{A}$ ). At $t>0$ after the quench $\mathbb{G}_{m, n}(t)$ is obtained as follows. One first defines $U_{k j} \equiv \sum_{m} R_{k m} e^{i E_{m} t}\left(R^{\dagger}\right)_{m j}$, where $R_{k j}$ is constructed as $R_{k j} \equiv\left\langle 0\left|c_{j}\right| v_{k}\right\rangle$ and $E_{m}$ is given by Eq. (A5). One then has

$$
\mathbb{G}(t)=U^{\dagger} \mathbb{G}(0) U \text {. }
$$

The explicit expression after performing the matrix multiplications in Eq. (C2) reads

$$
\begin{gathered}
\mathbb{G}_{m, n}(t)=\sum_{k, k^{\prime}} e^{-2 \pi i \frac{k}{L} m+2 \pi i \frac{k^{\prime}}{L} n+i\left(E_{k^{\prime}}-E_{k}\right) t}\left[\frac{1}{L} \delta_{k k^{\prime}}-\right. \\
\frac{1}{L^{2}} \frac{1}{L_{A}} \sum_{r=-k_{F}}^{k_{F}} \frac{1-e^{2 \pi i \omega k}}{1-e^{i \frac{2 \pi}{L_{A}}(r+\omega k)}} \times \frac{1-e^{-2 \pi i \omega k^{\prime}}}{\left.1-e^{-i \frac{2 \pi}{L_{A}}\left(r+\omega k^{\prime}\right)}\right] .}
\end{gathered}
$$

It is convenient to define the matrix $\mathbb{F}_{r, m}(t)$ as

$$
\mathbb{F}_{r, m}(t) \equiv \frac{1}{L} \sum_{k} e^{-2 \pi i \frac{k}{L} m-i E_{k} t} \frac{1-e^{2 \pi i \omega k}}{1-e^{i \frac{2 \pi}{L_{A}}(r+\omega k)}}
$$


Thus one can rewrite Eq. (C3) in the form

$$
\mathbb{G}_{m, n}(t)=\delta_{m, n}-\frac{1}{L_{A}} \sum_{r=-k_{F}}^{k_{F}} \mathbb{F}_{m, r}(t) \mathbb{F}_{n, r}^{*}(t) .
$$

Finally, the entanglement entropy for part $A$ of the chain after the geometric quench is obtained from the eigenvalues of Eq. (C5) restricted to $A$, using Eq. (B9).

\section{Appendix D: Dynamics after quenching to the infinite chain}

In this section we focus on the entanglement dynamics after a geometric quench in the limit $\omega \rightarrow 0$ (quench to the infinite chain, cf. Fig. 1). Notice that the limit $\omega \rightarrow 0$ is taken at fixed finite $L_{A}$.

The time evolved correlation function $\mathbb{G}_{m, n}(t)$ has the same form as in Eq. (C5) after redefining $\mathbb{F}_{m, r}(t)$ as

$$
\mathbb{F}_{m, r}(t)=\frac{1}{2 \pi} \int_{0}^{2 \pi} d k \frac{1-e^{i L_{A} k}}{1-e^{i \frac{2 \pi r}{L_{A}}+i k}} e^{-i m k+i t \cos k} .
$$

One should observe that in the limit $L_{A} \gg 1$, the second term in the numerator in Eq. (D1) is highly oscillating and one can write

$$
\begin{array}{r}
\mathbb{F}_{m, k_{s}}(t) \stackrel{L_{A} \gg 1}{\longrightarrow} \frac{1}{2 \pi} \mathcal{P} \int_{0}^{2 \pi} d k \frac{e^{-i m k+i t \cos k}}{1-e^{i\left(k+k_{s}\right)}}+ \\
\frac{1}{2} e^{i m k_{+} i t \cos k_{s}}
\end{array}
$$

where $\mathcal{P}$ denotes the Cauchy principal value of the integrand and we introduced $k_{s} \equiv 2 \pi r / L_{A}$. The approximation Eq. (D2) holds provided that $m \ll L_{A}, t \ll L_{A}$, and $k_{s} \neq 0$.

A numerically more convenient expression is obtained writing

$$
\mathbb{F}_{m, k_{s}}(t)=\sum_{p=0}^{L_{A}} e^{i p k_{s}+i(m-p) \frac{\pi}{2}} J_{p-m}(2 t),
$$

where $J_{q}(x)$ is the modified Bessel function. In the limit $L_{A} \rightarrow \infty$, using Eq. (C5), $k_{s}$ becomes a continuous variable and one can write $\mathbb{G}_{m, n}(t)$ as

$$
\mathbb{G}_{m, n}(t)=\delta_{m n}-\frac{1}{2 \pi} \int_{-\frac{\pi}{2}}^{\frac{\pi}{2}} d k_{s} \mathbb{F}_{m, k_{s}}(t) \mathbb{F}_{n, k_{s}}^{*}(t) .
$$

After using Eq. (D3) and performing explicitly the integration in Eq. (D4), $\mathbb{G}_{m, n}(t)$ reads

$$
\begin{aligned}
& \mathbb{G}_{m, n}(t)=\delta_{m n}- \\
& \sum_{p, q=0}^{L_{A}-1} \frac{\sin \left[\frac{1}{2} \pi(p-q)\right]}{\pi(p-q)} J_{p-m}(2 t) J_{q-n}^{*}(2 t) i^{m-p-n+q} .
\end{aligned}
$$

Notice that we keep $L_{A}$ finite in Eq. (D5), although formally the limit $L_{A} \rightarrow \infty$ is taken in Eq. (D4). It is convenient to redefine $s=(p+q) / 2$ and $d=(p-q) / 2$ obtaining

$$
\begin{aligned}
& \mathbb{G}_{m, n}(t)=\delta_{m n}- \\
& \sum_{d=-L_{A} / 2}^{L_{A} / 2} \sum_{s=|d|}^{L_{A}-|d|} \frac{\sin \pi d}{2 \pi d} J_{s+d-m}(2 t) J_{s-d-n}^{*}(2 t) i^{m-n-2 d} .
\end{aligned}
$$

Since $\sin (\pi d) / d$ is highly oscillating, in practice in Eq. (D5) it is possible to restrict the summation to the first few values of $d$. It is also worth reminding that $J_{q}(x)$ are exponentially vanishing at $|q|>x$, implying in Eq. (D6) that the contribution of the sum vanishes at $|s+d-m| / 2>t$ and $|s-d-n| / 2>t$, which is a manifestation of the Lieb-Robinson bound [127] in free models.

\section{Appendix E: Some remarkable scaling properties at large times}

In this section we derive the scaling form Eq. (22) for the von Neumann entropy at large times $t \rightarrow \infty$ after the quench from a finite to the infinite chain (cf. Appendix D).

The main ingredient of the calculation is the asymptotic behavior at $t \rightarrow \infty$ of the matrices $\mathbb{F}_{m, r}(t)$ (cf. Eq. (D1)). This is obtained using the stationary phase method [128]. The large-time behavior of $\mathbb{F}_{m, r}$ depends crucially on the parity of $L_{A}$ and on the value of $r$.

In particular, for $r=0$, irrespective of the parity of $L_{A}$, one obtains

$$
\mathbb{F}_{m, 0}(t) \approx \frac{\sqrt{2}}{\sqrt{\pi t}}\left[\frac{(-1)^{m}}{2} e^{i t-i \frac{\pi}{4}}+L_{A} e^{-i t+i \frac{\pi}{4}}\right],
$$

where we neglect terms $\mathcal{O}\left(t^{-1}\right)$. For generic $r \neq 0$ and odd $L_{A}$ one has

$$
\mathbb{F}_{m, r}(t) \approx(-1)^{m} \frac{e^{i t}}{\sqrt{4 \pi t}}\left[(1-i)-(1+i) \frac{\sin k_{s}}{1+\cos k_{s}}\right]
$$

with $k_{s}$ as in Eq. (D1). Finally, for $r \neq 0$ and even $L_{A}$ the result reads

$$
\begin{gathered}
\mathbb{F}_{m, r}(t) \approx \frac{L_{A}(-1)^{m}}{\sqrt{8 \pi t^{3}}}\left[i^{m}\left(2 m-L_{A}\right) \cos \left(t-m \frac{\pi}{2}+\frac{\pi}{4}\right)\right. \\
+\frac{1}{2}\left(2 m \tan \frac{k_{s}}{2}-L_{A} \tan \frac{k_{s}}{2}+i \sec ^{2} \frac{k_{s}}{2}\right) e^{i t-i \frac{\pi}{4}}- \\
\left.(-1)^{m} \frac{1}{4} \operatorname{cosec}^{2} \frac{k_{s}}{2}\left(2 i+\left(L_{A}-2 m\right) \sin k_{s}\right) e^{-i t+i \frac{\pi}{4}}\right]
\end{gathered}
$$

Notice that Eq. (E3) gives $\mathbb{F}_{m, r}(t) \sim t^{-3 / 2}$, which is subleading compared to Eqs. (E1) and (E2).

The corresponding asymptotic expansion for $\mathbb{G}_{m, n}(t)$ is straightforward, substituting Eqs. (E1)-(E3) into Eq. (C5). We start discussing the case with $L_{A}$ odd. The result reads

$$
\begin{array}{r}
\mathbb{G}_{m, n}(t)=\delta_{m, n}-\frac{(-1)^{m+n}}{\pi L_{A} t} \sum_{r=-k_{F}}^{k_{F}} \frac{1}{1+\cos \frac{2 \pi r}{L_{A}}} \\
+i \frac{e^{-2 i t}\left[(-1)^{m} e^{2 i t}+i L_{A}\right]\left[i(-1)^{n}+e^{2 i t} L_{A}\right]}{2 \pi L_{A} t} .
\end{array}
$$


It is convenient to rewrite Eq. (E4) as

$$
\begin{aligned}
& \mathbb{G}_{m, n}(t)=\delta_{m, n}+\frac{1}{t} u_{m} u_{n}\left[A(-1)^{m+n}+\right. \\
& \left.B(-1)^{m} e^{2 i t}+C(-1)^{n} e^{-2 i t}+D\right]
\end{aligned}
$$

introducing the constants $A, B, C, D$ as

$$
\begin{aligned}
A & \equiv-\frac{1}{\pi L_{A}} \sum_{r=-k_{F}}^{k_{F}} \frac{1}{1+\cos \frac{2 \pi r}{L_{A}}}-\frac{1}{2 \pi L_{A}} \\
B & =C^{*} \equiv \frac{i}{2 \pi} \\
D & =-\frac{L_{A}}{2 \pi} .
\end{aligned}
$$

In Eq. (E5) we defined $u_{m}$ as the vector of length $L_{A}$ with unit entries, i.e., $u_{m} \equiv(1,1, \ldots, 1)$. The eigenvalues of $\mathbb{G}_{m, n}(t)$ can be calculated analytically. It turns out that $\mathbb{G}$ has $L_{A}-2$ unit eigenvalues. Only two eigenvalues contribute non trivially to the entanglement entropy, which are given as

$$
\zeta_{ \pm}=1+\frac{A L_{A}+D L_{A} \pm L_{A} \sqrt{A^{2}+4 B C-2 A D+D^{2}}}{t} .
$$

Using Eq. (B9) and Eq. (E9), and considering the limit $1 \ll$ $L_{A} \ll t$, one obtains that the entropy is a scaling function of $t / L_{A}^{2}$, and can be given as

$$
S_{A}(t) \approx \frac{L_{A}^{2}}{2 \pi t}\left[1-\log \frac{L_{A}^{2}}{2 \pi t}\right]
$$

Clearly, since $S_{A}(t)>0 \forall t$, Eq. (E10) is valid only at $t \gg$ $L_{A}^{2}$.

We now turn to the case of $L_{A}$ even. The correlation matrix $\mathbb{G}_{m, n}(t)$, keeping only terms $\mathcal{O}\left(t^{-1}\right)$ is given as

$$
\begin{aligned}
\mathbb{G}_{m, n}(t) & =\delta_{m, n}+\frac{u_{m} u_{n}}{2 \pi L_{A} t} \times \\
& {\left[i(-1)^{m}-e^{-2 i t} L_{A}\right]\left[i(-1)^{n} e^{-2 i t}+L_{A}\right] . }
\end{aligned}
$$

A similar analysis as for odd $L_{A}$ gives the entanglement entropy as

$$
\begin{aligned}
S_{A}(t)=-\left[1-\frac{1+L_{A}^{2}}{2 \pi t}\right] & \log \left[1-\frac{1+L_{A}^{2}}{2 \pi t}\right] \\
& -\frac{1+L_{A}^{2}}{2 \pi t} \log \frac{1+L_{A}^{2}}{2 \pi t} .
\end{aligned}
$$

Notice that, as expected, in the limit $1 \ll L_{A}$ Eq. (E12) reduces to Eq. (E10).
[1] A. Polkovnikov, K. Sengupta, A. Silva, and M. Vengalattore, Rev. Mod. Phys. 83, 863 (2011).

[2] I. Bloch, J. Dalibard, and W. Zwerger, Rev. Mod. Phys. 80, 885 (2008).

[3] M. Rigol, V. Dunjko, and M. Olshanii, Nature 452, 854 (2008).

[4] M. Greiner, O. Mandel, T. W. Hänsch, and I. Bloch, Nature 419, 51 (2002).

[5] S. Hofferberth, I. Lesanovsky, B. Fischer, T. Schumm, and J. Schiedmayer, Nature 449, 324 (2007).

[6] S. Trotzky, Y.-A. Chen, A. Flesch, I. P. McCulloch, U. Schollwöck, J. Eisert, and I. Bloch, Nature Phys. 8, 325 (2012).

[7] M. Cheneau, P. Barmettler, D. Poletti, M. Endres, P. Schauss, T. Fukuhara, C. Gross, I. Bloch, C. Kollath, and S. Kuhr, Nature 481, 484 (2012).

[8] M. Gring, M. Kuhnert, T. Langen, T. Kitagawa, B. Rauer, M. Schreitl, I. Mazets, D. A. Smith, E. Demler, and J. Schmiedmayer, Science 337, 1318 (2012).

[9] F. Meinert, M. J. Mark, E. Kirilov, K. Lauber, P. Weinmann, A. J. Daley, and H.-C. Nägerl, Phys. Rev. Lett. 111, 053003 (2013).

[10] T. Kinoshita, T. Wenger, and D. S. Weiss, Nature 440, 900 (2006).

[11] U. Schneider, L. Hackermüller, J. P. Ronzheimer, S. Will, S. Braun, T. Best, I. Bloch, E. Demler, S. Mandt, D. Rasch, and A. Rosch, Nature Phys. 8, 213 (2012).

[12] J. P. Ronzheimer, M. Schreiber, S. Braun, S. S. Hodgman, S. Langer, I. P. McCulloch, F. Heidrich-Meisner, I. Bloch, and U. Schneider, Phys. Rev. Lett. 110, 205301 (2013).
[13] A. Reinhard, J.-F. Riou, L. Zundel D. S. Weiss, S. Li, A. M. Rey, and R. Hipolito, Phys. Rev. Lett. 110, 033001 (2013)

[14] C. Holzhey, F. Larsen, and F. Wilczek, Nucl. Phys. B 424, 443 (1994).

[15] G. Vidal, J. I. Latorre, E. Rico, and A. Kitaev, Phys. Rev. Lett. 90, 227902 (2003).

[16] J. I. Latorre, E. Rico, and G. Vidal, Quant. Inf. Comp. 4, 048 (2004).

[17] P. Calabrese and J. Cardy, J. Stat. Mech. (2004) P06002.

[18] L. Amico, R. Fazio, A. Osterloh, and V. Vedral, Rev. Mod. Phys. 80, 517 (2008).

[19] P. Calabrese, J. Cardy, and B. Doyon, J. Phys. A 42, 500301 (2009).

[20] J. Eisert, M. Cramer, and M. B. Plenio, Rev. Mod. Phys. 82, 277 (2010).

[21] P. Calabrese and J. Cardy, J. Stat. Mech. (2007) P10004,

[22] P. Calabrese and J. Cardy, J. Stat. Mech. (2007) P06008.

[23] J.-M. Stéphan and J. Dubail, J. Stat. Mech. (2011) P08019.

[24] V. Eisler and I. Peschel, J. Stat. Mech. (2007) P06005.

[25] V. Eisler, F. Iglói, and I. Peschel, J. Stat. Mech. (2009) P02011.

[26] M. Fagotti and P. Calabrese, Phys. Rev. A 78, 010306 (2008).

[27] F. Iglói, Z. Szatmari, and Y.-C. Lin, Phys. Rev. B 80, 024405 (2009).

[28] B. Hsu, E. Grosfeld, and E. Fradkin, Phys. Rev. B 80, 235412 (2009).

[29] U. Divakaran, F. Iglói, and H. Rieger, J. Stat. Mech. (2011) P10027.

[30] F. Iglói, Z. Szatmari, and Y.-C. Lin, Phys. Rev. B 85, 094417 (2012). 
[31] K. Schönhammer, Phys. Rev. B 75, 205329 (2007).

[32] G. C. Levine, M. J. Bantegui, and J. A. Burg, Phys. Rev. B 86, 174202 (2012).

[33] T. Sabetta and G. Misguich, Phys. Rev. B 88, 245114 (2013).

[34] V. Eisler and Z. Rácz, Phys. Rev. Lett. 110, 060602 (2013).

[35] M. Collura, S. Sotiriadis, and P. Calabrese, J. Stat. Mech. (2013) P09025.

[36] L. Bucciantini, M. Kormos, and P. Calabrese, J. Phys. A: Math. Theor. 47, 175002 (2014).

[37] G. De Chiara, S. Montangero, P. Calabrese, and R. Fazio, J. Stat. Mech. (2006) P03001.

[38] A. Läuchli and C. Kollath, J. Stat. Mech. (2008) P05018.

[39] V. Eisler and I. Peschel, EPL 99, 20001 (2012).

[40] H. Kim and D. A. Huse, Phys. Rev. Lett. 111, 127205 (2013).

[41] M. Collura and P. Calabrese, J. Phys. A: Math. Theor. 46, 175001 (2013)

[42] P. Hauke and L. Tagliacozzo, Phys. Rev. Lett. 111, 207202 (2013)

[43] J. Schachenmayer, B. P. Lanyon, C. F. Roos, and A. J. Daley, Phys. Rev. X 3, 031015 (2013).

[44] S. Kessler, I. P. McCulloch, and F. Marquardt, New J. Phys. 15, 053043 (2013)

[45] M. Collura, M. Kormos, and P. Calabrese, J. Stat. Mech. (2014) P01009.

[46] A. Zamora, J. Rodriguez-Laguna, M. Lewenstein, and L. Tagliacozzo, arXiv:1401.7916.

[47] P. Calabrese and J. Cardy, J. Stat. Mech. (2005) P04010.

[48] A. J. Daley, H. Pichler, J. Schachenmayer, and P. Zoller, Phys. Rev. Lett. 109, 020505 (2012).

[49] D. A. Abanin and E. Demler, Phys. Rev. Lett. 109, 020504 (2012).

[50] J. Mossel, G. Palacios, and J.-S. Caux, J. Stat. Mech. L09001 (2010).

[51] T. Kinoshita, T. Wenger, and D. S. Weiss, Science 305, 1125 (2004).

[52] M. Rigol and A. Muramatsu, Phys. Rev. Lett. 94, 240403 (2005).

[53] M. Rigol and A. Muramatsu, Phys. Rev. Lett. 93, 230404 (2004).

[54] M. Rigol and A. Muramatsu, Mod. Phys. Lett. B 19, 861 (2005).

[55] A. Minguzzi and D. M. Gangardt, Phys. Rev. Lett. 94, 240404 (2005).

[56] D. M. Gangardt and M. Pustilnik, Phys. Rev. A 77, 041604 (2008).

[57] F. Heidrich-Meisner, M. Rigol, A. Muramatsu, A. E. Feiguin, and E. Dagotto, Phys. Rev. A 78, 013620 (2008).

[58] F. Heidrich-Meisner, S. R. Manmana, M. Rigol, A. Muramatsu, A. E. Feiguin, and E. Dagotto, Phys. Rev. A 80, 041603(R) (2009).

[59] S. Langer, M. J. A. Schuetz, I. P. McCulloch, U. Schollwöck, and F. Heidrich-Meisner, Phys. Rev. A 85, 043618 (2012).

[60] L. Vidmar, S. Langer, I. P. McCulloch, U. Schneider, U. Schollwöck, and F. Heidrich-Meisner, Phys. Rev. B 88, 235117 (2013).

[61] J. Kajala, F. Massel, and P. Törmä, Phys. Rev. Lett. 106, 206401 (2011)

[62] T. Antal, Z. Rácz, A. Rákos, and G. M. Schütz, Phys. Rev. E 59, 4912 (1999).

[63] D. Karevski, Eur. J. Phys. B 27, 147 (2002).

[64] D. Gobert, C. Kollath, U. Schollwöck, and G. Schütz, Phys. Rev. E 71, 036102 (2005).

[65] R. Steinigeweg, J. Gemmer, and M. Michel, EPL 75, 75406 (2006).
[66] L. Santos, Phys. Rev. E 78, 031125 (2008).

[67] M. Haque, Phys. Rev. A 82, 012108 (2010).

[68] J. Lancaster and A. Mitra, Phys. Rev. E 81, 06134 (2010).

[69] J. Lancaster, E. Gull, and A. Mitra, Phys. Rev. B 82, 235124 (2010).

[70] J. Mossel and J.-S. Caux, New J. Phys. 12, 055028 (2010).

[71] S. Jesenko and M. Znidaric, Phys. Rev. B 84, 174438 (2011).

[72] L. Santos and A. Mitra, Phys. Rev. E 84, 016206 (2011).

[73] J. C. Halimeh, A Wöllert, I. P. McCulloch, U. Schollwöck, and T. Barthel, Phys. Rev. A 89, 063603 (2014).

[74] X. Zotos, F. Naef, and P. Prelovšek, Phys. Rev. B 55, 11029 (1997).

[75] F. Heidrich-Meisner, A. Honecker, and W. Brenig, Eur. Phys. J. Spec. Topics 151, 135 (2007).

[76] S. R. White, Phys. Rev. Lett. 69, 2863 (1992).

[77] U. Schollwöck, Rev. Mod. Phys. 77, 259 (2005).

[78] U. Schollwöck, Annals of Physics 326, 96 (2011).

[79] S. Langer, F. Heidrich-Meisner, J. Gemmer, I. P. McCulloch, and U. Schollwöck, Phys. Rev. B 79, 214409 (2009).

[80] S. Langer, M. Heyl, I. P. McCulloch, and F. Heidrich-Meisner, Phys. Rev. B 84, 205115 (2011).

[81] R. Steinigeweg, S. Langer, F. Heidrich-Meisner, I. P. McCulloch, and W. Brenig, Phys. Rev. Lett. 106, 160602 (2011).

[82] M. Ganahl, E. Rabel, F. H. L. Essler, and H. G. Evertz, Phys. Rev. Lett. 108, 077206 (2012).

[83] T. Fukuhara, P. Schausz, M. Endres, S. Hild, M. Cheneau, I. Bloch, and C. Gross, Nature 502, 76 (2013).

[84] T. Fukuhara, A. Kantian, M. Endres, M. Cheneau, P. Schausz, S. Hild, D. Bellem, U. Schollwöck, T. Giamarchi, C. Gross, I. Bloch, S. Kuhr, Nature 9, 235 (2013)

[85] V. Zauner, M. Ganahl, H. G. Evertz, and T. Nishino, arXiv:1207.0862.

[86] C. Karrasch, J. E. Moore, and F. Heidrich-Meisner, Phys. Rev. B 89, 075139 (2014).

[87] F. Iglói, G. Roós, and L. Turban, J. Stat. Mech. (2014) P03023.

[88] T. Antal, Z. Rácz, and L. Sasvári, Phys. Rev. Lett. 78, 167 (1997).

[89] M. Rigol, V. Dunjko, V. Yurovsky, and M. Olshanii, Phys. Rev. Lett. 98, 50405 (2007).

[90] P. Calabrese, M. Mintchev, and E. Vicari, Phys. Rev. Lett. 107, 020601 (2011).

[91] P. Calabrese, M. Mintchev, and E. Vicari, J. Stat. Mech. (2011) P09028.

[92] E. Vicari, Phys. Rev. A 85, 062324 (2012).

[93] A. J. Daley, C. Kollath, U. Schollwöck, and G. Vidal, J. Stat. Mech. (2004) P04005.

[94] S. White and A. Feiguin, Phys. Rev. Lett. 93, 266402 (2004).

[95] H.-J. Mikeska and A. K. Kolezhuk, Lecture Notes in Physics 645, 1 (2004).

[96] H. W. J. Blöthe, J. Cardy, and M. P. Nightingale, Phys. Rev. Lett. 56, 742 (1986).

[97] I. Affleck, Phys. Rev. Lett. 56, 746 (1986).

[98] P. di Francesco, P. Mathieu, and D. Senechal, Conformal field theory, Springer (1997).

[99] P. Ginsparg, Fields, Strings and Critical Phenomena, Les Houches, Session XLIX, 1988 ed. by E. Brézin and J. ZinnJustin, (1989).

[100] V. Eisler, D. Karevski, T. Platini, and I. Peschel, J. Stat. Mech. (2008) P01023.

[101] I. Peschel and V. Eisler, J. Phys. A: Math. Theor. 42, 504003 (2009).

[102] T. Antal, P. L. Krapivsky, and A. Rákos, Phys. Rev. E 78, 061115 (2008).

[103] F. Iglói, G. Roósz, and Y.-C. Lin, New J. Phys. 15, 023036 
(2013).

[104] P. Calabrese and J. Cardy, Phys. Rev. Lett. 96, 136801 (2006).

[105] V. Eisler and I. Peschel, J. Stat. Mech. (2014) P04005.

[106] I. Peschel, M. Kaulke, and O. Legeza, Ann. Physik (Lipzig) 8, 153 (1999).

[107] I. Peschel and M.-C. Chung, J. Phys. A 32, 8419 (1999).

[108] M.-C. Chung and I. Peschel, Phys. Rev. B 64, 064412 (2001).

[109] I. Peschel, J. Phys. A 36, L205 (2003).

[110] I. Peschel, J. Stat. Mech. (2004) P06004.

[111] S. Langer, Diploma thesis, RWTH Aachen 2009.

[112] J. Des Cloizeaux and M. Gaudin, J. Math. Phys. 7, 1384 (1966).

[113] E. Lieb, T. Schultz, and D. Mattis, Ann. Phys. 16, 407 (1961).

[114] E. Barouch, B. M. McCoy, and M. Dresden, Phys. Rev. A 2, 1075 (1970).

[115] E. Barouch and B. McCoy, Phys. Rev. A 3, 786 (1971).

[116] E. Barouch and B. M. McCoy, Phys. Rev. A 3, 2137 (1971).

[117] B. M. McCoy, E. Barouch, and D. B. Abraham, Phys. Rev. A
4, 2331 (1971).

[118] J. I. Latorre and A. Riera, J. Phys. A 42, 504002 (2009).

[119] M. Fagotti and P. Calabrese, J. Stat. Mech. (2010) P04016.

[120] V. Alba, L. Tagliacozzo, and P. Calabrese, Phys. Rev. B 81, 060411(R) (2010).

[121] V. Alba, L. Tagliacozzo, and P. Calabrese, J. Stat. Mech. (2011) P06012.

[122] F. Iglói and I. Peschel, Europhys. Lett. 89, 40001 (2010).

[123] M. Fagotti and P. Calabrese, J. Stat. Mech. (2011) P01017.

[124] M. Fagotti, EPL 97, 17007 (2012).

[125] J. H. H. Perk and H. W. Capel, Physica A 89, 265 (1977).

[126] J. H. H. Perk and H. Au-Yang, J. Stat. Phys. 135, 599 (2009).

[127] E. H. Lieb and D. W. Robinson, Commun. Math. Phys. 28, 251 (1972).

[128] A. Erdélyi, Asymptotic Expansions, New York: Dover Publications (1956). 